(Prof. Dr. med G. Hasenfuß)

Im Zentrum Innere Medizin

Der Medizinischen Fakultät der Universität Göttingen

\title{
Prädiktoren für die Prognose nach perkutaner Mitralklappenreparatur mit dem MitraClip ${ }^{\circledR}$-System
}

\author{
INAUGURAL-DISSERTATION \\ zur Erlangung des Doktorgrades \\ der Medizinischen Fakultät der \\ Georg-August-Universität zu Göttingen
}

\author{
vorgelegt von \\ Tobias Tichelbäcker
}

aus

Kempen

Göttingen 2014 
Dekan:

1. Berichterstatter:

2. Berichterstatter/in:

3. Berichterstatter/in:
Prof. Dr. rer. nat. H. K. Kroemer

Prof. Dr. med. W. Schillinger

PD Dr. med. Theodoros Tirilomis

PD Dr. med. Matthias Sigler

Tag der mündlichen Prüfung: $\quad$ 23.09.2014 


\section{Inhaltsverzeichnis}

\section{Abkürzungsverzeichnis}

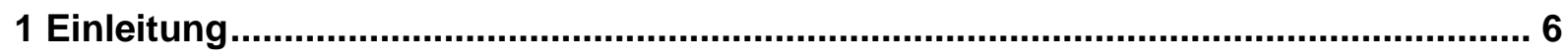

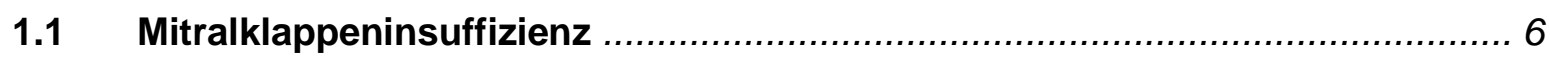

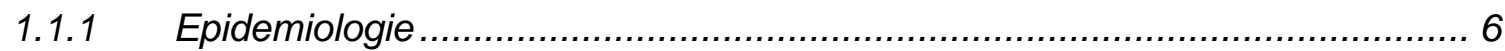

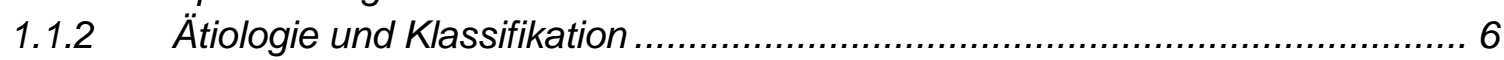

1.1.3 Pathophysiologie der chronischen Mitralinsuffizienz .................................. 8

1.1.4 Prognose der Mitralinsuffizienz ............................................................ 9

1.1.5 Therapie der Mitralinsuffizienz ............................................................ 10

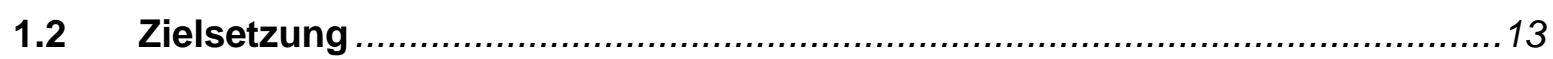

2 Material und Methoden .........................................................................................14

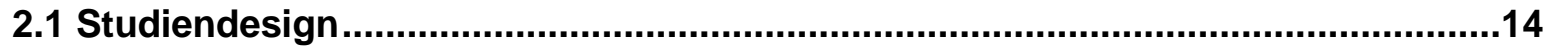

2.2 Patientenselektion und präinterventionelle Diagnostik ......................................14

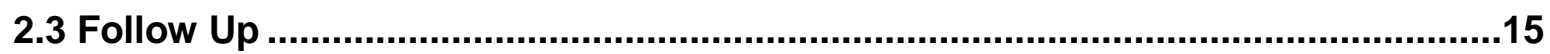

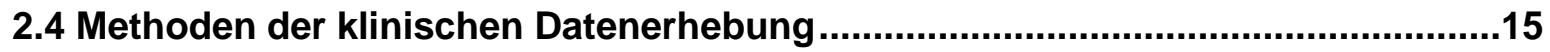

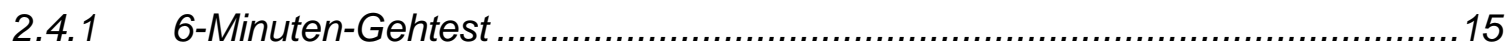

2.4.2 Minnesota Living With Heart Failure Questionnaire (MLHFQ) .......................16

2.4.3 New York Heart Association (NYHA)-Klassifikation ................................... 16

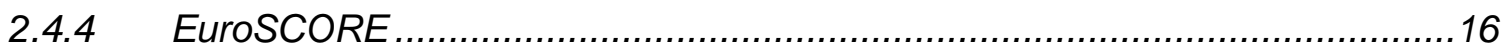

2.4.5 STS (Society Of Thoracic Surgeons) Score ...........................................17

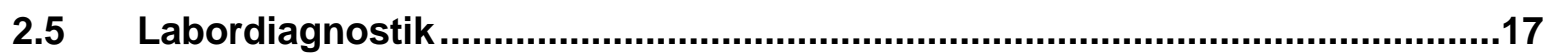

2.6 Echokardiographie ..................................................................................18

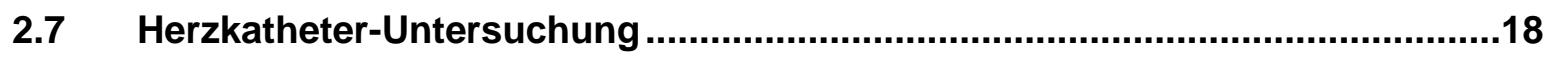

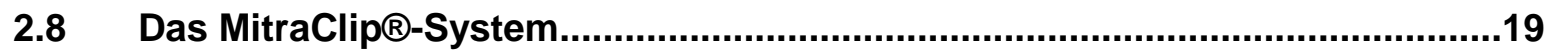

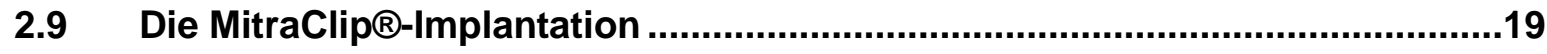

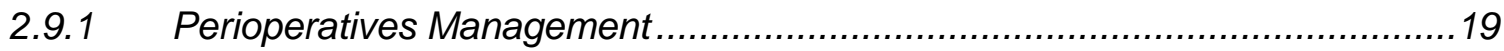

2.9.2 Ablauf der perkutanen Mitralklappenreparatur ........................................20

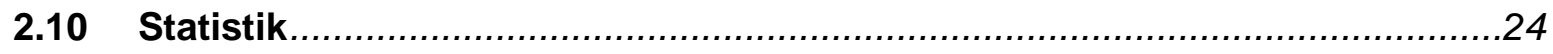

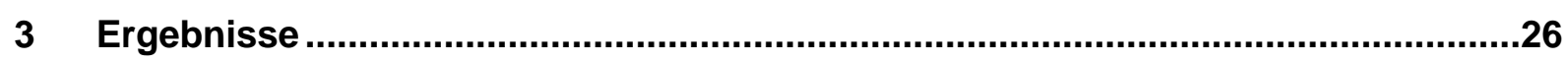

3.1 Basisdemographische Charakteristika der Göttinger MitraClip®-Kohorte.....26

3.2 Sicherheit der Intervention und prozedurale Ergebnisse ..............................28

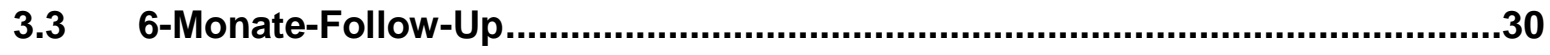

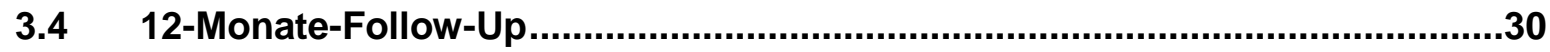

3.5 Kaplan-Meier-Analyse Überleben ...........................................................33

3.6 Kaplan-Meier-Analyse Hospitalisierung wegen Herzinsuffizienz ....................36 
3.7 Herzinsuffizienzbedingte Hospitalisierungsrate vor und nach Intervention...39

3.8 Prädiktoren des akuten prozeduralen Versagens ..........................................40

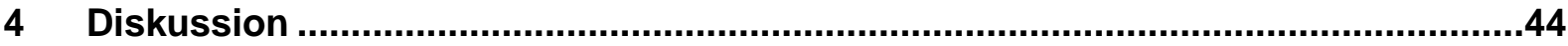

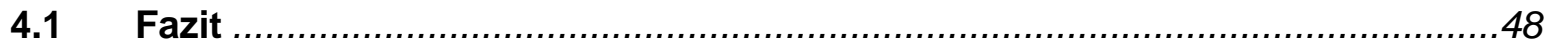

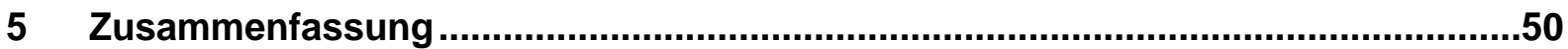

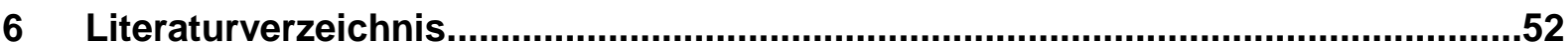

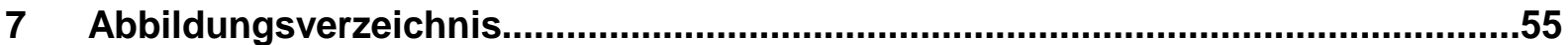

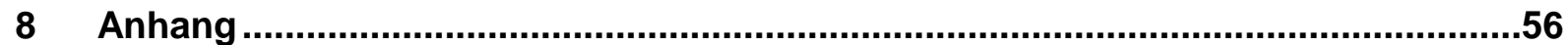

8.1 Minnesota Living With Heart Failure Questionnaire.........................................56

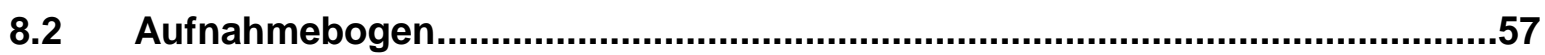




\section{Abkürzungsverzeichnis}

APS

Akuter prozeduraler Erfolg (âcute procedural șuccess)

ASS

Acetylsalicylsäure

COPD

Chronisch obstruktive Lungenerkrankung

CRT-D

Cardiale Resynchronisation-Therapie - (and) Defibrillation

DCM

Dilatative Kardiomyopathie

ECLIA

Enhanced Chemiluminescence Immunoassay System

ESC

European Society of Cardiology

ICM Ischämische Kardiomyopathie

INR International Normalized Ratio

LVEDD Linksventrikulärer enddiastolischer Diameter

LVEDV Linksventrikuläres enddiastolisches Volumen

LV-EF Linksventrikuläre Ejektionsfraktion

LVESD Linksventrikulärer endsystolischer Diameter

LVESV Linksventrikuläres endsystolisches Volumen

MLHFQ Minnesota Living with Heart Failure Questionnaire

MI Mitralinsuffizienz

NYHA New York Heart Association

PAVK Periphere arterielle Verschlusskrankheit

SOP Standard Operating Procedures

STS Society of Thoracic Surgeons

TEE Transösophageale Echokardiographie

TTE Transthorakale Echokardiographie 


\section{EINLEITUNG}

\subsection{MitralkLapPeninsuffiZIENZ}

Die Mitralklappe befindet sich zwischen dem linken Vorhof und dem linken Ventrikel. Während der Systole ist sie geschlossen und verhindert so den Rückstrom des Blutes aus der Kammer in den Vorhof. Die Undichtigkeit der Mitralklappe wird als Mitralklappeninsuffizienz bezeichnet.

\subsubsection{EPIDEMIOLOGIE}

Die Mitralklappeninsuffizienz ist nach der Aortenklappenstenose die zweithäufigste behandlungsbedürftige Herzklappenerkrankung in Europa (lung et al. 2003). Im Euro Heart Survey wurden 2003 insgesamt 5001 Patienten aus 25 europäischen Ländern eingeschlossen. Bei ungefähr einem Drittel (31,5\%, 877 Patienten) der nicht bereits operierten Patienten war im Rahmen dieser Studie eine Mitralinsuffizienz festgestellt worden. In den USA geht man von schätzungsweise über zwei Millionen betroffenen Patienten aus (Nkomo et al. 2006).

\subsection{2 ÄTIOLOGIE UND KLASSIFIKATION}

Grundsätzlich kann man die Mitralinsuffizienz in akut und chronisch unterteilen. Die akute Mitralklappeninsuffizienz tritt am häufigsten nach Myokardinfarkt mit konsekutivem Papillarmuskelabriss oder als Folge einer Endokarditis auf und sollte chirurgisch korrigiert werden (Vahanian et al. 2012). Im Vordergrund dieser Arbeit soll aber die chronische Mitralinsuffizienz stehen, die man getrennt nach Ursache und Mechanismus, folgendermaßen unterteilt (vgl. Abb 1. und 2.).

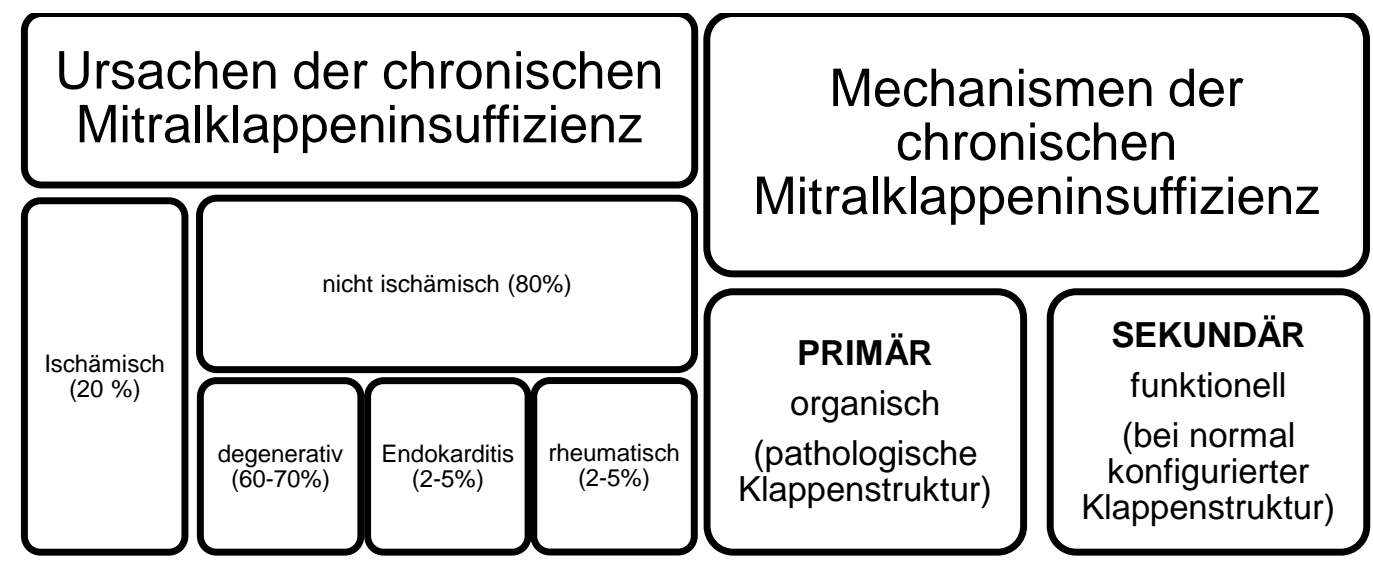

Abbildung 1

Abbildung 2

Abb.1+2: Ursachen und Mechanismen der chronischen Mitralinsuffizienz (nach Enriquez-Sarano et al. 2009) 
Die Ursachen der chronischen Mitralinsuffizienz unterteilt man in solche ischämischen und solche nicht-ischämischen Ursprungs. Bei der ischämischen Mitralinsuffizienz stehen die Auswirkungen der koronaren Herzkrankheit im Vordergrund. Durch beispielsweise myokardiale Narben nach Myokardinfarkt können die Papillarmuskeln des Klappenhalteapparats der Mitralklappe erheblich in ihrer Funktion gestört werden. Dies kann über die Chorda tendinae zu einem erhöhten Zug auf die Segel der Mitralklappe und somit zu einer unzureichenden Schlussfähigkeit der Klappe führen (Enriquez-Sarano et al. 2009).

Die nicht-ischämischen Ursachen der Mitralinsuffizienz sind vielfältig. Den größten Anteil machen aktuell in Europa aufgrund der zunehmenden Anzahl an älteren Patienten die degenerativen Erkrankungen der Mitralklappe aus. Die Bandbreite dieser Erkrankungen reicht vom Prolaps eines der Segel bis zur Kalzifizierung der Segel oder des Annulus. In der Vergangenheit war das rheumatische Fieber noch führende Ursache der Mitralinsuffizienz, bevor die Prävalenz der Krankheit in Europa immer mehr abgenommen hat. Auch eine bakterielle Endokarditis kann eine Destruktion der Mitralklappe und somit eine Insuffizienz verursachen.

Zusammenfassend muss man allerdings feststellen, dass die Zuordnung einer manifesten Mitralinsuffizienz zu genau einer Ursache oft nicht zweifelsfrei gelingt und häufig Mischbilder vorliegen.

Bei den Mechanismen der chronischen Mitralinsuffizienz wird anhand der Klappenmorphologie zwischen primär und sekundär unterschieden. Bei einer pathologischen Veränderung der Klappensegel selbst (z.B. bei einem Segelprolaps oder infolge einer Endokarditis) spricht man von einer primären Mitralinsuffizienz. Dagegen sind die Mitralsegel bei einer sekundären Mitralinsuffizienz vollkommen intakt, aber infolge einer linksventrikulären Dilatation (bei dilatativer Kardiomyopathie) oder eines lokalen linksventrikulären Remodelings (nach Myokardinfarkt) kommt es zu einer Verlagerung der Papillarmuskeln und damit zu einem Zug (tethering) über die Chordae tendineae an den Segeln (vgl. Abb. 3). Dieses führt zu einer Verlagerung der Segelkoaptation in den linken Ventrikel hinein und damit zu einer Verminderung der Koaptationsfläche, wodurch es zu einer Klappeninsuffizienz kommt. 


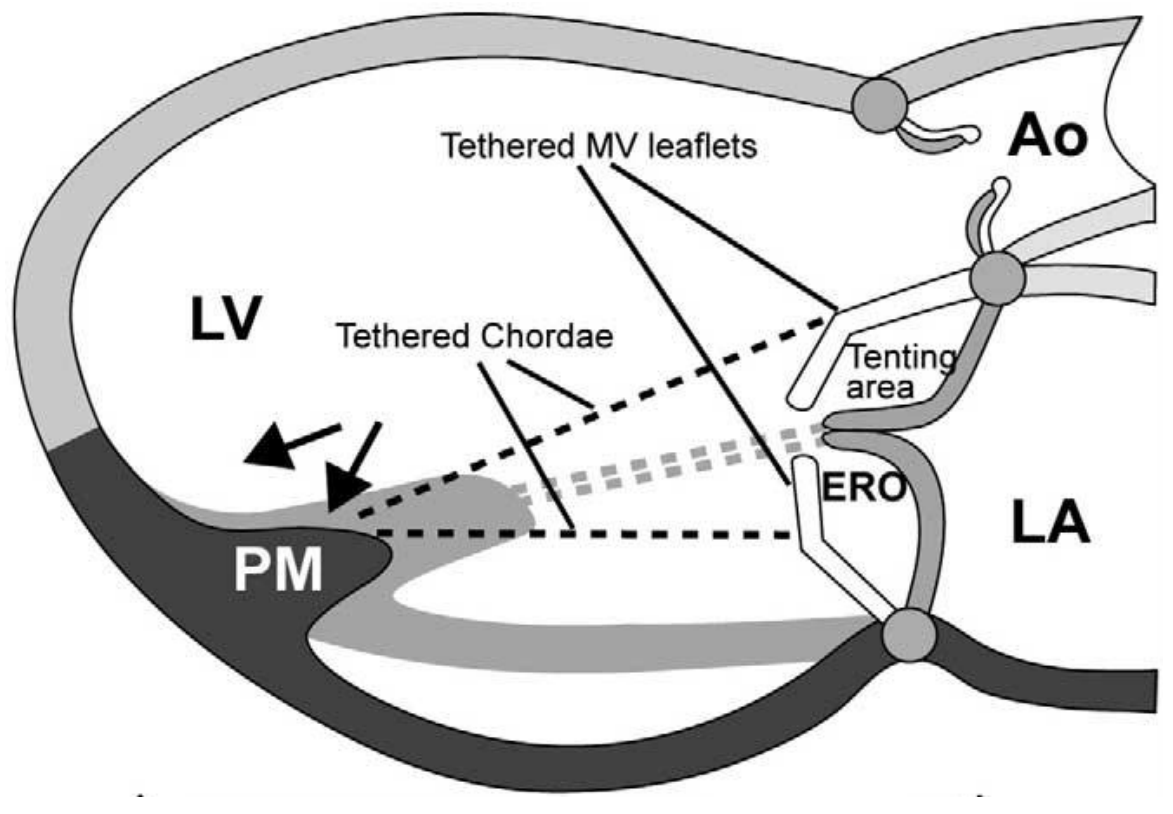

Abbildung 3: tethering-Mechanismus (aus: Bursi et al. 2006, S.105)

Für die chirurgische, aber auch für die interventionelle Therapie spielen sowohl die Ursache als auch der Mechanismus der Mitralinsuffizienz eine entscheidende Rolle (Carpentier 1983).

\subsubsection{PATHOPHYSIOLOGIE DER CHRONISCHEN MITRALINSUFFIZIENZ}

Das Regurgitationsvolumen, das während der Systole vom linken Ventrikel über die insuffiziente Mitralklappe in den linken Vorhof zurückströmt, führt zu einer erhöhten Vorlast des linken Herzens. Das Regurgitationsvolumen ist abhängig von der Größe der Klappenöffnung und dem Druckgradienten über der Mitralklappe (Erdmann 2011; Camm et al. 2006). Die chronische Volumenbelastung des linken Herzens bewirkt langfristig eine Dilatation des linken Ventrikels, die anfänglich durch linksventrikuläre Hypertrophie des linken Ventrikels noch kompensiert werden kann. Die erhöhte Wandspannung, die nach dem Gesetz von Laplace nun nötig ist, um das gleiche Volumen bei größerem Ventrikeldiameter auszuwerfen, schädigt aber das linksventrikuläre Myokard. Allerdings kann die Pumpfunktion infolge der erhöhten Vorlast (Frank-Starling-Mechanismus) anfangs sogar ansteigen und auch lange grenzwertig stabil bleiben, so dass sie kein geeigneter Parameter zur Abschätzung kardialen Remodelings ist. Vielmehr sollte hierfür der linksventrikuläre endsystolische Diameter (LVESD) gemessen werden (Enriquez-Sarano et al. 2009). Die Annulusdilatation kann die 
bestehende Mitralinsuffizienz erhöhen und so den klinischen Zustand und die Prognose des Patienten weiter verschlechtern (Grigioni et al. 2001).

Durch die erhöhte Volumenbelastung wird nicht nur der linke Ventrikel, sondern auch das linke Atrium dilatiert, was als Mechanismus für die Entstehung von Vorhofflimmern diskutiert wird (Camm et al. 2010). Weiterhin kann die Volumenbelastung des linken Herzens langfristig zu einer pulmonalen Hypertonie führen (Erdmann 2011; Camm et al. 2006).

\subsubsection{Prognose Der Mitralinsuffizienz}

Bei der Prognose der Mitralinsuffizienz sollte man nach den verschiedenen Ätiologien der Mitralinsuffizienz unterscheiden.

Für die primäre Mitralinsuffizienz gilt, dass eine vorhandene Symptomatik und der Schweregrad der Mitralinsuffizienz sowie das Alter wichtige Prädiktoren für das Überleben und das Auftreten kardialer Ereignisse sind. So berichten Enriquez - Sarano et al. (Enriquez-Sarano et al. 2009) über kardiale Ereignisraten von 10-12\% pro Jahr bei Patienten mit schwerer Mitralinsuffizienz und Mortalitätsraten von $0,8 \%$ oder $12 \%$ pro Jahr bei asymptomatischen beziehungsweise symptomatischen Patienten unter medikamentöser Therapie. Weitere Parameter, die mit einem schlechten Outcome bei primärer Mitralinsuffizienz einhergingen, waren Vorhofflimmern, pulmonale Hypertension, geringe LV-EF, Dilatation des linken Vorhofs sowie ein erhöhter LVESD.

Bei der sekundären Mitralinsuffizienz ist die Prognose deutlich schlechter, da hier auch schon geringe Grade der Mitralinsuffizienz zu einer deutlich schlechteren Lebenserwartung führen (s. Abb. 4). Eine koronare Herzkrankheit sowie eine eingeschränkte linksventrikuläre Pumpfunktion sind weitere wichtige Einflussfaktoren. Allerdings ist hier aufgrund der limitierten Datenlage unklar, ob tatsächlich die Mitralinsuffizienz der Grund für die schlechte Prognose ist oder doch eher die Grunderkrankung, die auch zur Mitralinsuffizienz führte (Vahanian et al. 2012). 


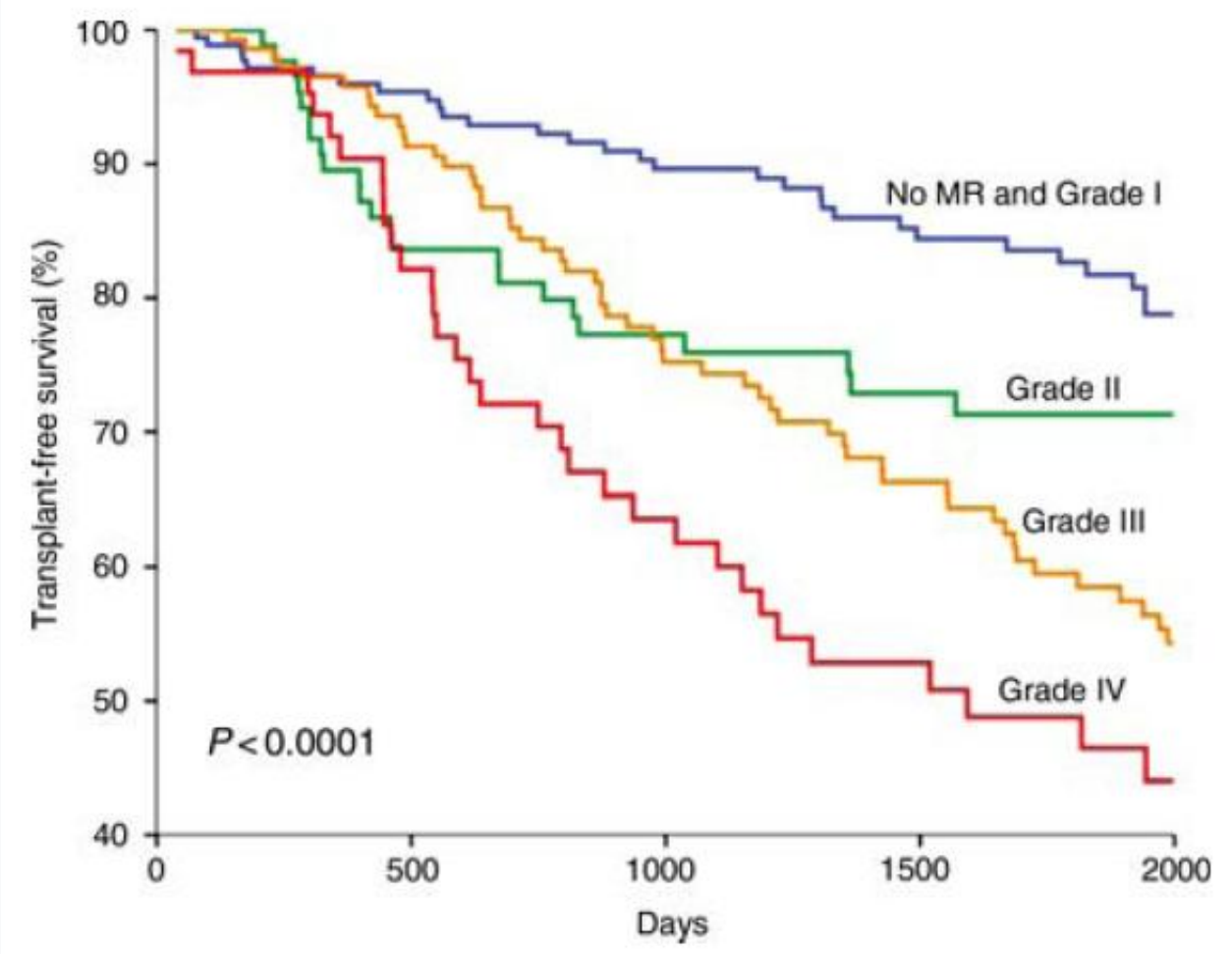

Abbildung 4: Kaplan-Meier-Analyse der Lebenserwartung von Patienten nach Myokardinfarkt mit unterschiedlichen Schweregraden einer ischämischen Mitralinsuffizienz (aus: Bursi et al. 2010, S.385)

\subsubsection{THERAPIE DER MITRALINSUFFIZIENZ}

Grundsätzlich gibt es drei verschiedene Möglichkeiten, Patienten mit einer Mitralinsuffizienz zu behandeln.

Die medikamentöse Therapie beinhaltet die Senkung der Nachlast durch Vasodilatation der peripheren arteriellen Gefäße, um so das Regurgitationsvolumen möglichst klein zu halten. Mit der gleichen Intention können auch Diuretika zur Senkung der Vorlast gegeben werden. Vor allem bei einer akut aufgetretenen Mitralinsuffizienz, nach chirurgischer Therapie und weiterhin bestehender Symptomatik sowie bei Patienten, die nicht für eine chirurgische Therapie in Frage kommen, wird diese medikamentöse Therapie angewendet (Vahanian et al. 2012).

In den aktuellen Leitlinien der European Society of Cardiology (Vahanian et al. 2012) wird festgestellt, dass die chirurgische Therapie der Goldstandard zur Behandlung der primären Mitralinsuffizienz ist. Bei der sekundären Mitralinsuffizienz ist die Empfehlung dagegen weniger gut belegt. Bei Patienten mit bestehender Indikation zur operativen Revaskularisation 
aufgrund einer signifikanten koronaren Herzerkrankung soll aber bei höhergradiger Mitralinsuffizienz zum Zeitpunkt der Bypass-Operation ein Mitralklappenrekonstruktion durchgeführt werden, insbesondere wenn die linksventrikuläre Funktion erhalten ist.

Für die chirurgische Behandlung der Mitralinsuffizienz gibt es sehr unterschiedliche Konzepte. Zunächst unterscheidet man zwischen dem Mitralklappenersatz und der Mitralklappenreparatur. Hier ist die vorherrschende Meinung, dass die Mitralklappenreparatur deutliche Vorteile gegenüber dem Ersatz bietet und, soweit durchführbar, die zu bevorzugende Therapie ist (Vahanian et al. 2012). Die Vorteile einer Reparatur liegen vor allem in der Vermeidung der Implantation von Fremdmaterial und damit der Vermeidung einer dauerhaften therapeutischen Antikoagulation sowie im Erhalt des physiologischen Klappenapparates. Die Mortalität scheint nach erfolgreicher operativer Mitralklappenreparatur deutlich geringer zu sein als nach Mitralklappenersatz, es gibt allerdings keine randomisierten Studien zu dieser Frage (Glower 2012).

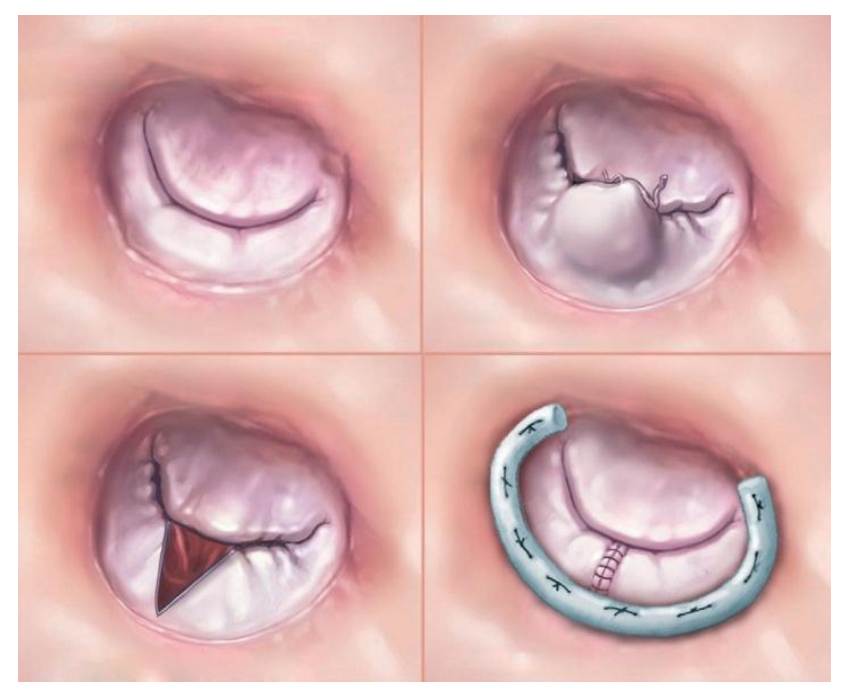

Abbildung 5: oben links: normale Mitralklappe; oben rechts: Prolaps posteriores Segel; unten links: trianguläre Resektion des prolabierten Segelanteils; unten rechts: Endergebnis des operierten Segels und Versorgung mit einem Annuloplastiering (aus: Enriquez-Sarano et al. 2009 S.1383)

Entscheidet sich der Operateur für die Reparatur der Klappe, stehen ihm auch hier verschiedene Möglichkeiten zur Verfügung. So werden bei einem Prolaps des posterioren Segels die entsprechenden Anteile des Segels entfernt und die beiden Schnittenden wieder miteinander verbunden (Glower 2012). Ein weiteres Reparaturverfahren zur Behandlung der Mitralinsuffizienz ist die Annuloplastie, bei der der Mitralring mittels einer kleineren starren Ringprothese verkleinert wird, um so die Mitralklappenöffnungsfläche insgesamt zu verringern (Vahanian et al. 2012). Oft wird dieses Verfahren auch mit anderen Reparaturverfahren der 
Mitralklappe kombiniert, da Studien zeigen, dass so die Rezidivrate der Mitralinsuffizienz nach Operation deutlich gesenkt werden konnte (Cohn et al. 1994).

Ein weiteres chirurgisches Verfahren, auf dem auch das Prinzip des MitraClip®-Systems beruht, ist die sogenannte Alfieri-Naht. 1992 versorgte der italienische Herzchirurg Alfieri seinen ersten Patienten mit dieser „edge-to-edge“-Reparatur, indem er die Ränder des anterioren und posterioren Mitralsegels zentral auf einem Abschnitt von drei bis fünf Millimetern mit einer Naht verband, so dass zwei Öffnungen resultierten, um so die Mitralinsuffizienz zu verringern (Glower 2012).

Die alternative Option zur Behandlung der Mitralinsuffizienz bei ausgewählten Patienten ist die perkutane Mitralklappenreparatur mit dem MitraClip®-System der Firma Abbott Vascular (Abbott Park, Illinois, USA). Bei diesem Verfahren werden mit Hilfe eines Clips, der nach venöser Leistenpunktion transseptal in das linke Herz vorgebracht wird, die beiden Segel der Mitralklappe nach dem Prinzip der Alfieri-Naht miteinander verbunden. Seit 2008 ist das System mit CE - Kennzeichnung auch in Deutschland verfügbar und wird an mittlerweile über 80 deutschen Zentren angewendet. Weltweit wurden über 9000 Patienten mit dem MitraClip®System behandelt (Mitteilung Abbott Vascular).

Bezüglich der perkutanen Mitralklappenreparatur mittels MitraClip® liegen inzwischen robuste Daten vor, die die Sicherheit und Wirksamkeit dieses Verfahrens belegen. In der EVEREST-IStudie (Feldman et al. 2009) konnte gezeigt werden, dass die Mitralklappenreparatur mit dem MitraClip $\AA$-System sicher und praktikabel ist. In dieser Studie wurden insgesamt 107 Patienten an 31 Zentren in den USA behandelt. Ein zufriedenstellendes Ergebnis (Mitralinsuffizienz $\leq 2+$ ) wurde in $74 \%$ der Fälle erreicht. Während der Prozedur verstarb keiner der Patienten und nur ein Patient verstarb post-interventionell im Verlauf des gleichen Aufenthaltes.

Die EVEREST-II-Studie (Feldman et al. 2011) war die erste randomisierte Studie, in der die perkutane Mitralklappenreparatur mit der chirurgischen Therapie verglichen wurde. Es wurden insgesamt 279 Patienten mit moderater (Grad 3) bis schwerer (Grad 4) symptomatischer Mitralinsuffizienz in einem Verhältnis von 2:1 (perkutane Mitralklappenreparatur $n=184$; Chirurgie $n=95$ ) eingeschlossen. Nur 27\% der Patienten hatten eine funktionelle Ätiologie der Mitralinsuffizienz. Im Vergleich war die perkutane Therapie zwar weniger effizient in der Reduktion der Mitralinsuffizienz, wies jedoch eine geringere Komplikationsrate innerhalb der ersten 30 Tage nach Eingriff auf (15\% vs. 48\% Major Adverse Events), was hauptsächlich an der selteneren Indikation zur Bluttransfusionen in der perkutanen Gruppe lag. Während des Follow-up-Zeitraumes mussten sich allerdings $20 \%$ der primär interventionell behandelten 
Patienten aufgrund einer signifikanten residualen Mitralinsuffizienz einem chirurgischen Mitralklappeneingriff unterziehen. Die Mortalität im Follow-up betrug 6\% in beiden Gruppen. Eine Subgruppenanalyse implizierte, dass die MitraClip®-Prozedur bei Patienten mit einem Alter > 70 Jahren, einer funktionellen Mitralinsuffizienz und einer eingeschränkten linksventrikulären Ejektionsfraktion $(<60 \%)$ der chirurgischen Therapie hinsichtlich der Effizienz nicht unterlegen sei. Randomisierte kontrollierte Studien zu dieser Fragestellung stehen allerdings noch aus.

Das europäische ACCESS-EU-Register mit 14 teilnehmenden Zentren und 567 deutlich älteren und kränkeren Patienten bildet den aktuellen Stand der Mitra-Clip-Therapie in der alltäglichen klinischen Routine großer europäischer Zentren ab. Auch für diese Hochrisikopatienten konnte die Sicherheit und Wirksamkeit der Prozedur gezeigt werden (Maisano et al. 2013).

\subsection{ZIELSETZUNG}

Es ist nach wie vor unklar, welche Subgruppen an Patienten am meisten von einer MitraClip®Therapie profitieren. Ziel der vorliegenden Arbeit ist es daher, mögliche Prädiktoren für das Outcome nach perkutaner Mitralklappenreparatur mit dem MitraClip®-System in einem Kollektiv von Hochrisikopatienten zu identifizieren, denen aufgrund ihrer Komorbiditäten und gesundheitlichen Verfassung eine chirurgische Therapie verwehrt geblieben wäre. Untersucht wurde dafür die Göttinger MitraClip®-Kohorte von 150 konsekutiven Patienten, die im Zeitraum von April 2009 bis Juni 2012 im Herzzentrum der Universitätsmedizin Göttingen nach Indikationsstellung durch das Heart Team mit MitraClip® behandelt wurden. 


\section{Material Und Methoden}

\subsection{STUDIENDESIGN}

Wir führten eine prospektive Verlaufsbeobachtung der ersten 150 Patienten durch, die zwischen April 2009 und Juni 2012 im Herzzentrum der Universitätsmedizin Göttingen mittels MitraClipß behandelt wurden. Das positive Votum der Göttinger Ethikkommission lag vor und alle Patienten gaben ihre schriftliche Einwilligung zur Teilnahme an dieser Studie sowie zur Durchführung der MitraClipß-Implantation.

\subsection{Patientenselektion Und PRÄINTERVENTIONeLle DiagnostiK}

Die Patienten, die aufgrund ihrer symptomatischen Mitralinsuffizienz für die perkutane Mitralklappenreparatur mit dem MitraClip®-System in Frage kamen, wurden im Vorfeld nach Erhebung der Anamnese mit Hilfe von transösophagealer und transthorakaler Echokardiographie (TEE bzw. TTE) untersucht. Weiterhin wurden eine Koronarangiographie und eine LV-Angiographie durchgeführt. Anschließend wurden die Befunde der Patienten im „Heart Team“ (interdisziplinäre Konferenz bestehend aus interventionellen Kardiologen und Herzchirurgen) besprochen, das auf Grundlage aktueller Leitlinien (Vahanian et al. 2012) Indikation und Verfahren zur Behandlung signifikanter Mitralklappeninsuffizienzen überprüfte und festlegte.

Eingeschlossen wurden Patienten, die mindestens 18 Jahre alt waren, unter einer signifikanten Mitralklappeninsuffizienz litten und ein hohes Risiko für eine konventionelle herzchirurgische Operation aufwiesen. Im Gegensatz dazu waren eine floride Endokarditis oder eine planimetrische Mitralklappenöffnungsfläche $<4 \mathrm{~cm}^{2}$ Ausschlusskriterien für die Behandlung mit MitraClip ${ }^{\circledR}$.

Gleichzeitig wurde nochmals eine spezielle Anamnese erhoben, bei der vor allem die Krankengeschichte der letzten 12 Monate sowie der aktuelle Gesundheitsstatus im Fokus standen. Weiterhin wurde vor dem Eingriff der standardisierte 6-Minuten-Gehtest durchgeführt, die Vitalparameter gemessen, sowie das NT-proBNP im Blut des Patienten bestimmt.

Prozedurassoziierte Parameter wie Länge des Eingriffes, Durchleuchtungszeit oder Dauer der Intubation wurden erhoben. 
Kurz vor der Entlassung der Patienten wurden diese wieder mittels Echokardiographie untersucht, die Vitalparameter und das NT-proBNP bestimmt.

\subsection{FOLLOW UP}

Die Follow-Up-Untersuchungen der Patienten erfolgte nach $6 \pm 2$ und $12 \pm 2$ Monaten hauptsächlich in der Abteilung Kardiologie der Universitätsmedizin Göttingen. Bei einigen Patienten war dies aber aufgrund der großen Distanz zu ihrem Wohnort nicht möglich, so dass in diesen Fällen die örtlichen betreuenden Kardiologen um Durchführung der Untersuchungen gebeten wurden. Während der Follow-Up-Untersuchungen wurden bei den Patienten wiederum die Anamnese und die klinische Untersuchung durchgeführt, die Vitalparameter erhoben, das NTproBNP bestimmt und der 6-Minuten-Gehtest sowie die Echokardiographie wiederholt.

Im Zeitraum von Juni bis Juli 2012 wurden alle Patienten, soweit möglich, telefonisch kontaktiert und über ihren aktuellen Gesundheitszustand und eventuelle Krankenhausaufenthalte seit der MitraClip®-Implantation befragt. Außerdem wurden die Hausärzte der 150 Patienten gebeten, alle Berichte über kardiologische Krankenhausaufenthalte im Zeitraum von zwei Jahren vor MitraClip®-Implantation bis Juli 2012 den Untersuchern der Studie zur Verfügung zu stellen.

\subsection{Methoden DeR KLinischen Datenerhebung}

Im Folgenden sollen die einzelnen Methoden der klinischen Datenerhebung aufgelistet und näher erläutert werden.

\subsubsection{6-MINUTEN-GEHTEST}

Der 6-Minuten-Gehtest wird als Parameter genutzt, um die Leistungsfähigkeit der Patienten vor und nach perkutaner Mitralklappenreparatur zu beurteilen. Es wird die Gehstrecke in Metern gemessen, die die Patienten innerhalb von sechs Minuten auf einer ebenen Strecke zurücklegen können. Die wenigsten Patienten gehen bei diesem Test an ihre maximalen Leistungsgrenzen, da die Patienten zum Beispiel zwischendurch auch pausieren dürfen. Dieser Test simuliert vielmehr die körperliche Leistungsfähigkeit der Patienten in ihrem persönlichen Alltag (American Thoracic Society 2002). Der Test wurde vor der MitraClipßImplantation sowie bei den Follow-Up-Untersuchungen nach sechs und 12 Monaten durchgeführt. 


\subsubsection{Minnesota LiVING With HEART FAILURE QuestionnaIRE (MLHFQ)}

Der Minnesota Living With Heart Failure Questionnaire (MLHFQ) wurde entwickelt, um die Lebensqualität von Patienten anhand eines Punktesystems zu erfassen. Der Test registriert dabei mit Hilfe von 21 standardisierten Fragen den körperlichen und psychischen Zustand des Patienten innerhalb der letzten vier Wochen. Pro Frage können 0-5 Punkte je nach Symptomatik vergeben werden. Mittlerweile ist der MLHFQ der am häufigsten durchgeführte Test für Lebensqualität in Studien zu pharmakologischen, chirurgischen und interventionellen Eingriffen bei Patienten mit Herzinsuffizienz (Morgan et al. 2007). Der MLHFQ - Fragebogen (s. Anhang 1) wurde von den Patienten vor dem Eingriff sowie bei den Follow-Up Untersuchungen nach sechs und 12 Monaten ausgefüllt.

\subsubsection{NEW YORK HEART ASSOCIATION (NYHA)-KLASSIFIKATION}

Die New York Heart Association-Klassifikation der Herzinsuffizienz umfasst vier Stufen, die wie folgt aufgegliedert sind: NYHA I beschreibt die Herzinsuffizienz ohne Symptomatik im Alltag; NYHA II - nur Symptome bei größerer Anstrengung; NYHA III - Symptome schon bei geringer körperlicher Belastung und NYHA IV - Symptomatik in Ruhe. Der NYHA-Status wurde ebenfalls zu den drei Zeitpunkten Baseline (vor Intervention), 6- und 12-MonatsFollow-Up Untersuchung erhoben.

\subsubsection{EUROSCORE}

Anhand des EuroSCORE kann mit Hilfe prädefinierter mathematischer Algorithmen ein Schätzwert für die 30-Tages-Mortalität eines Patienten nach einer herzchirurgischen Operation ermittelt werden. Dafür müssen verschiedene Parameter (z.B. Alter, Nierenfunktion, linksventrikuläre Funktion) in eine über das Internet erreichbare Maske eingegeben werden. Der Rechner ermittelt anhand der eingegebenen Werte dann das statistische perioperative Mortalitätsrisiko (http://www.euroscore.org/calc.html). Seit 2011 ist eine neue Version des EuroSCOREs verfügbar (EuroSCORE II). Die von uns benutzten Werte sind allerdings noch nach logistischem EuroSCORE I berechnet, der als verlässliches Instrument gilt, um die 30Tages-Mortalität nach herzchirurgischen Operationen vorherzusagen (Geissler et al. 2000). Dieser wurde beispielsweise auch im europäischen Register ACCESS Europe verwendet 
(Maisano et al. 2013), so dass eine Vergleichbarkeit gewährleistet ist. Der EuroSCORE wurde jeweils vor den Interventionen berechnet.

\subsubsection{STS (SOCIETY OF THORACIC SURGEONS) SCORE}

Der STS Score stellt ein weiteres Instrument zu Abschätzung des peri-operativen Mortalitätsrisikos herzchirurgischer Eingriffe dar, der im Hinblick auf isolierte konventionelle Herzklappenchirurgie eine höhere Genauigkeit besitzt als der EuroSCORE (Wendt et al. 2009). Auch bei diesem Risikorechner kann man durch die Eingabe von Parametern des Patienten das Mortalitätsrisiko ermitteln. In unserer Verlaufsbeobachtung wurde der STS Score jeweils vor der MitraClip®-Implantation ermittelt.

\subsection{LABORDIAGNOSTIK}

Ein weiterer Bestandteil der Baseline- sowie Follow-Up - Diagnostik war die Untersuchung der Blutwerte. Es wurden neben dem kleinen Blutbild (Hämoglobin, Hämatokrit, Erythrozyten, Thrombozyten, Leukozyten, Mean Corpuscular Volume (MCV), Mean Corpuscular Hemoglobin $(\mathrm{MCH})$, Mean Corpuscular Hemoglobin Concentration (MCHC)) das Kreatinin und das N-terminale pro Brain natriuretische Peptid (NT-proBNP) bestimmt.

Das NT-proBNP wird im Göttinger Zentrallabor aus dem Heparinplasma des Patienten gewonnen und mittels ECLIA (Enhanced Chemiluminescence Immunoassay System) bestimmt (Deutsche Akkreditierungsstelle 2011). Das NT-proBNP ist ein Hormon und wird in den Myozyten der Vorhöfe physiologisch auf einen Dehnungsreiz hin gebildet. Wenn das Herz entweder durch erhöhte Vorlast oder Nachlast dauerhaft belastet wird, bilden auch die Ventrikelmyozyten dieses Hormon (Schmidt 2007), und der Serumspiegel ist erhöht. NTproBNP kann so als Marker und Parameter für Verlaufskontrollen bei der chronischen Herzinsuffizienz genutzt werden (Maisel et al. 2008). 


\subsection{ECHOKARDIOGRAPHIE}

Die echokardiographischen Untersuchungen im Herzzentrum Göttingen wurden mit Hilfe eines Vivid E9 von der Firma GE (Fairfield, Connecticut) (Screening und Follow-up-Untersuchungen) und eines ie33 der Firma Philips (Amsterdam, Niederlande) (MitraClip®-Prozeduren) durchgeführt.

Alle Patienten wurden vor der MitraClip®-Implantation mit Hilfe einer transösophagealen Echokardiographie (TEE) und einer transthorakalen Echokardiographie (TTE) untersucht. Außerdem wurde das Behandlungsergebnis vor Entlassung beziehungsweise mindestens 24 Stunden nach dem Eingriff echokardiographisch kontrolliert. Bei den anschließenden FollowUp- Untersuchungen wurden die Patienten routinemäßig nur noch transthorakal echokardiographiert. Parameter, die während aller Follow-up-Untersuchungen kontrolliert wurden, waren der Schweregrad der Mitralinsuffizienz, der mittlere Gradient über der Mitralklappe (zur Evaluation des Stenosegrades), die Diameter des linken Ventrikels (LVEDD, LVESD) und des linken Vorhofs, die LV-EF sowie die linksventrikulären Volumina (LVEDV, LVESV). Die Quantifizierung der Mitralinsuffizienz erfolgte dabei, wie in der EVEREST-IStudie vorgeschlagen (Feldman et al., 2009), mittels einer Einteilung in 4 Schweregrade. Nach MitraClip®-Implantation wurden die von Foster (Foster et al. 2007) vorgeschlagenen Kriterien zur Einstufung zugrunde gelegt.

Der akute prozedurale Erfolg (APS, acute procedural success) der MitraClip®-Implantation war (wie in der Everest-II-Studie) definiert als residuale Mitralinsuffizienz des Schweregrades 2 oder weniger zum Zeitpunkt der Entlassung des Patienten. Um einen Einfluss der Vollnarkose auszuschließen, wurde explizit nicht der direkt postoperative MI-Schweregrad gewertet.

\subsection{HERZKATHETER-UNTERSUCHUNG}

Ein weiterer obligatorischer Teil des Screeningverfahrens vor MitraClip $\AA$ war die HerzkatheterUntersuchung. Ziel dieser Untersuchung ist es, den aktuellen Zustand der Koronargefäße zu kontrollieren und die Ätiologie der Mitralinsuffizienz zu klären, um damit die Diagnose der Mitralinsuffizienz zu komplettieren (Vahanian et al. 2012). Weiterhin kann eine Laevokardiographie durchgeführt werden, mit der eine Quantifizierung der Mitralinsuffizienz möglich 
ist, wenn zum Beispiel nicht-invasive Verfahren keine eindeutigen Ergebnisse liefern konnten (Hamm et al. 2008).

\subsection{Das MitraCliPß-SYSTEM}

Das MitraClip®-System besteht aus zwei großen Bestandteilen: Zum einem dem steuerbaren Führungskatheter, zum anderen dem Clip-Einführsystem. Der steuerbare Führungskatheter mit einem Außendurchmesser von 8,1 mm (24 French) dient nach der transseptalen Punktion als Zugangsweg für das Clip-Einführsystem und stellt so eine direkte Verbindung zwischen Punktionsstelle und linkem Vorhof dar. Außerdem ist der Führungskatheter Bestandteil der Steuerung des Clips. Das Clip-Einführsystem bringt an seiner Spitze den Clip bis zur angedachten Position im linken Atrium vor, während es am anderen Ende von den Händen des Untersuchers bedient werden kann. Der Clip kann, sobald er in das linke Atrium vorgeschoben wurde, auf allen drei Raumachsen gesteuert und so in die optimale Position zur Verringerung der Mitralinsuffizienz gebracht werden. Das MitraClip®-Implantat selbst besteht aus einer Metalllegierung und einem Polyestergewebe (evalve 2011).

\subsection{Die MitraClipß-IMPLANTATION}

\subsubsection{Perioperatives MANAGEMent}

Alle Patienten erhielten vor der Operation einen Harnblasenkatheter. Direkt vor Beginn der Operation wurden vom Anästhesisten jeweils ein zentraler Venenkatheter, ein peripherer Venenzugang und eine invasive arterielle Blutdruckmessung angelegt, ebenso erfolgte eine endotracheale Intubation. In aller Regel erfolgt die Extubation des Patienten unmittelbar nach Beendigung der Prozedur noch auf dem Kathetertisch. Die Apparatur zur arteriellen Blutdruckmessung wurde unmittelbar im Anschluss an die Intervention, die restlichen Zugänge, soweit nicht weiter benötigt, nach 24 Stunden entfernt. Der Druckverband wurde regelhaft 12 Stunden nach Eingriff abgenommen. Das Nahtmaterial (Z-Naht) der Inzision wurde nach 10 Tagen entfernt.

Patienten ohne Indikation für eine therapeutische Antikoagulation bekamen am Tag vor dem Eingriff ASS $100 \mathrm{mg}$. Grundsätzlich sollte auch bei diesen Patienten darauf geachtet werden, dass der INR unter 1,7 und der Quickwert über 50\% liegt. Nach der Intervention erhielten 
diese Patienten einmalig $300 \mathrm{mg}$ Clopidogrel, danach $75 \mathrm{mg}$ täglich für vier Wochen. ASS, soweit nicht schon verordnet, wird routinemäßig für sechs Monate jeweils $100 \mathrm{mg}$ täglich nach perkutaner Mitralklappenreparatur gegeben. Bei Patienten unter Antikoagulation mit einem Vitamin-K-Antagonisten wird die Antikoagulation im Vorfeld des Eingriffes auf Heparin umgestellt und am Operationstag pausiert. Nach der Intervention wird zusätzlich zum VitaminK-Antagonisten ASS 100mg für vier Wochen empfohlen. Bei allen Patienten wird im direkten Anschluss an die Intervention eine Thromboseprophylaxe durchgeführt (Schillinger und Kerl 2011).

\subsubsection{ABLAUf DER PERKUTANEN MITRALKLAPPENREPARATUR}

Alle Eingriffe wurden im Herzkatheterlabor der Universitätsmedizin Göttingen unter Vollnarkose des Patienten und in Anwesenheit eines Facharztes für Anästhesie wie bereits vorbeschrieben durchgeführt (Feldman et al. 2009, vgl. Abb. 6).
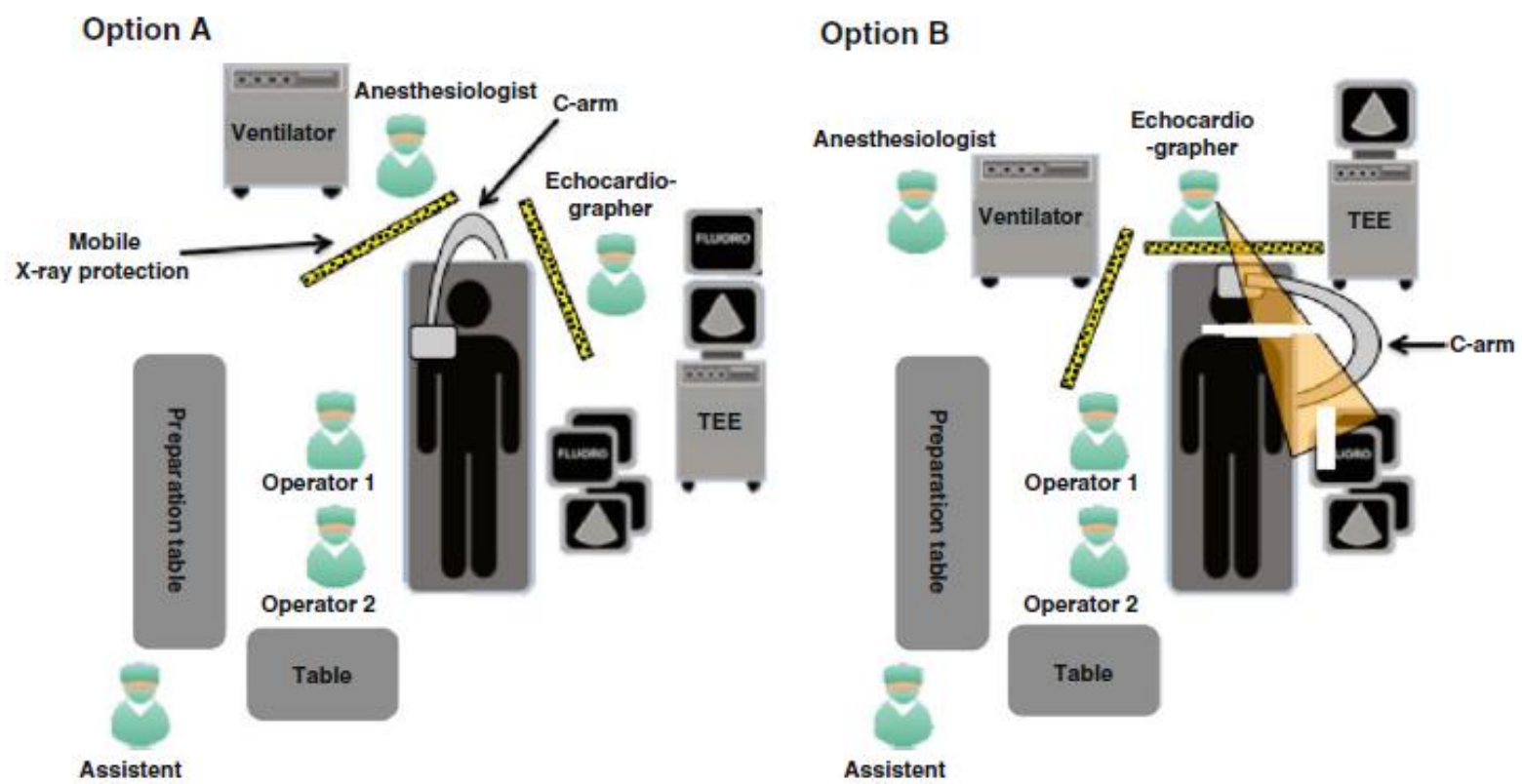

Abbildung 6: Lokales Setting der MitraClip®-Prozedur (Boekstegers et al. 2013)

Noch vor Beginn des eigentlichen Eingriffes erfolgt im Hybrid-OP eine nochmalige echokardiographische Befundkontrolle mittels TEE. Dabei wird die Anatomie der Mitralklappe dreidimensional dargestellt (vgl. Abb. 7 und 8). 

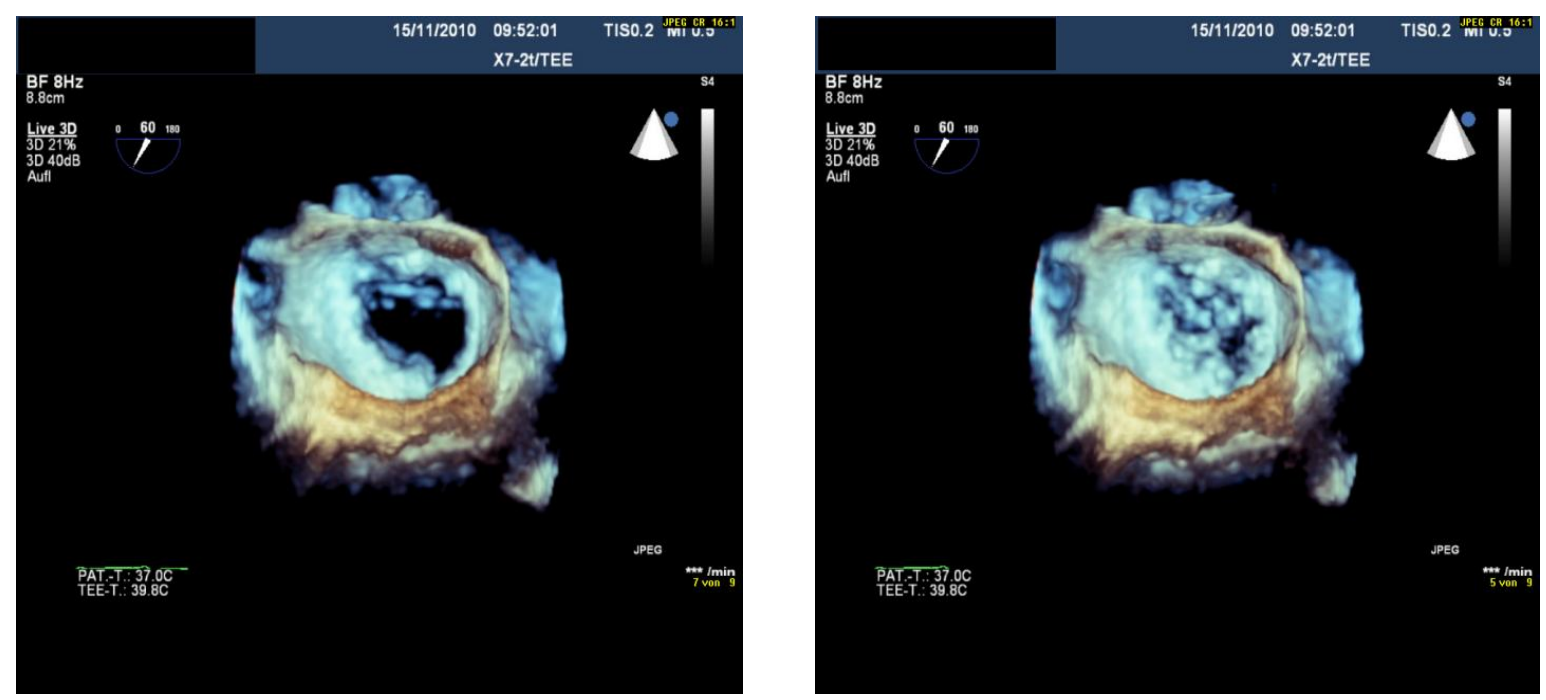

Abbildung 7: 3D-TEE, Aufsicht auf die Mitralklappe in der Diastole (Abbildungen 7-14 UMG, Klinik für

Abbildung 8: 3D-TEE, Aufsicht auf die Mitralklappe Kardiologie, Prof.Hasenfuß)

Genauer Ursprung sowie Schweregrad des Mitralinsuffizienzjets werden mittels Farbdoppler erneut evaluiert (Abb. 9).

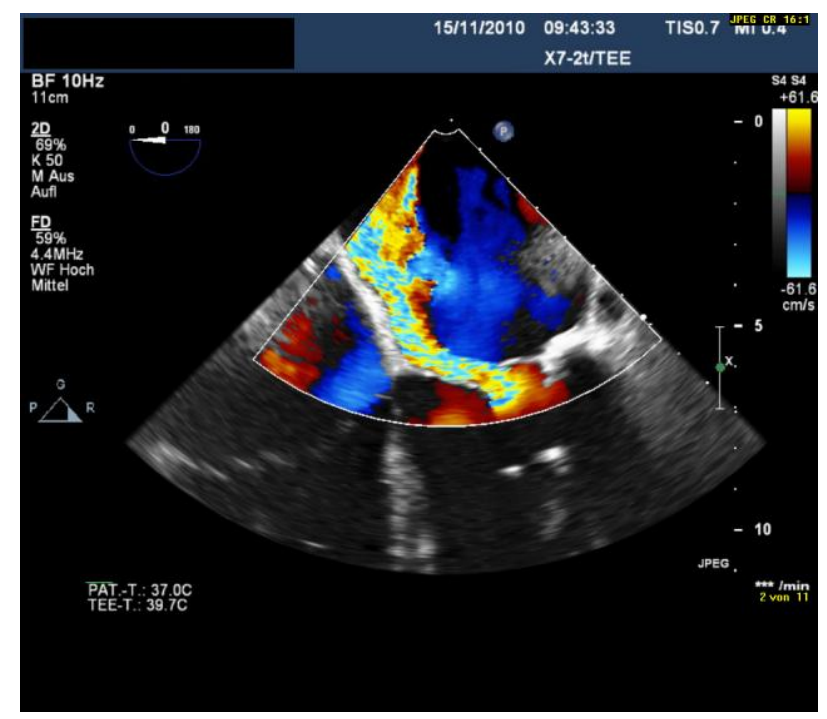

Abbildung 9: Deutlich sichtbarer exzentrischer Jet an der Wand des linken Vorhofs

Nach Punktion der Vena femoralis und Vorschieben der Transseptalnadel erfolgt die transseptale Punktion dann unter genauer echokardiographischer Kontrolle. Erst nachdem diese komplikationslos erfolgt ist, wird eine Vollheparinisierung des Patienten durchgeführt. 
Im Anschluss an die transseptale Punktion und die Heparinisierung des Patienten wird ein Dilatator über einen extrasteifen Führungsdraht vorgeschoben und die Punktionsstelle der Vena femoralis für den steuerbaren Führungskatheter (24 French Durchmesser am femoralen Zugang) gedehnt. Darauffolgend wird der steuerbare Führungskatheter mitsamt Dilatator unter radiologischer und echokardiographischer Kontrolle in den linken Vorhof vorgeschoben. Sobald der Führungskatheter sich sicher mit circa drei Zentimeter im linken Vorhof befindet, werden der Dilatator sowie der Führungsdraht zurückgezogen. Bei dem Vor-und Zurückschieben von MitraClip ${ }^{\circledR}$ System-Einheiten durch das Lumen des Führungskatheters muss immer auf die Entlüftung des Lumens geachtet werden, um Luftembolien zu vermeiden. Nun wird das Clip-Einführsystem über den steuerbaren Führungskatheter unter Durchleuchtung eingeführt und vorgeschoben. Wenn der Clip mit Hilfe von transösophagealer Echokardiographie im linken Vorhof sichtbar wird, sollte sichergestellt werden, dass dieser sich nicht an der linken Vorhofwand oder in unmittelbarer Klappennähe befindet. Kann dies ausgeschlossen werden, versucht man den Clip für den Greifversuch optimal zu positionieren: Dies bedeutet, dass eine Höhe von mindestens zehn Millimeter über den Klappensegeln erreicht, der Clip zentral über dem Insuffizienzjet positioniert und eine senkrecht axiale Ausrichtung zur Mitralklappenebene angestrebt werden sollte (vgl. Abb. 10+11).

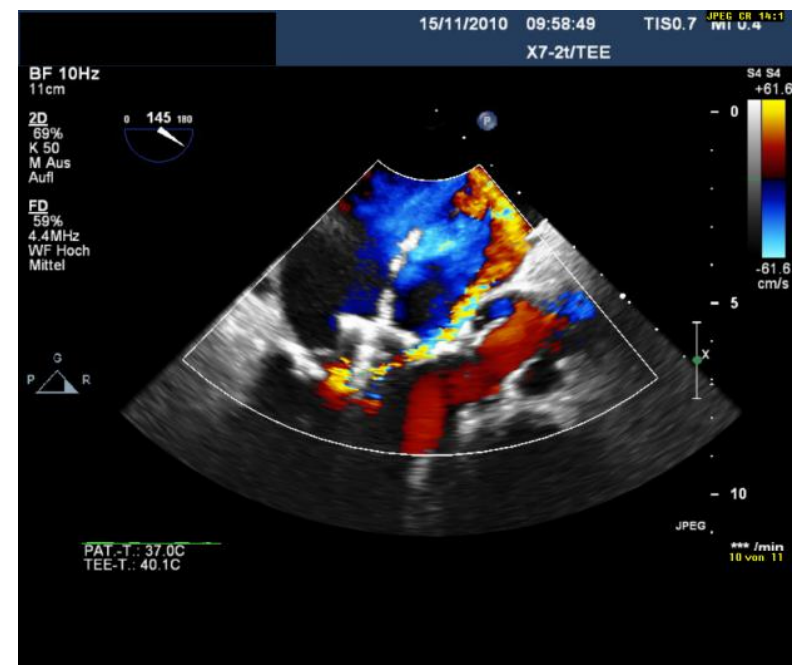

Abbildung 10

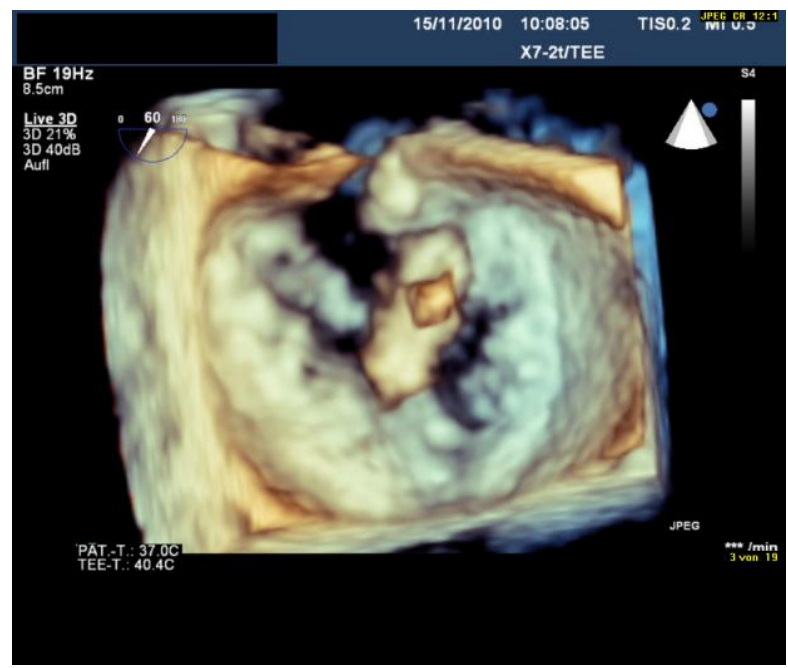

Abbildung 11

Platzierung und Ausrichtung des geöffneten MitraClip® an der Stelle des Regurgitationsjets mit Hilfe von 2D TEE (Abb.10) und 3D TEE (Abb.11)

Sind diese Voraussetzungen für den Untersucher in befriedigender Weise erfüllt, kann er die Arme des Clips bis auf einen Winkel von fast $180^{\circ}$ öfnen und die Greifer anheben. Alle diese Handlungen werden noch im linken Vorhof durchgeführt, um das Risiko zu vermindern, mit 
dem Clip Klappenstrukturen zu verletzten. Dann sollte das Clip-Einführsystem weiter vorgeschoben werden, bis der Clip zwei Zentimeter unterhalb der Mitralklappe im Ventrikel positioniert ist. Danach erfolgt die Anhebung der Clip-Arme auf einen Winkel von etwa $120^{\circ}$, dem sogenannten Greifarmwinkel. Sehr vorsichtig wird jetzt das Clip-Einführsystem zurückgezogen, um Anteile des posterioren sowie des anterioren Klappensegels zu greifen. Um zu kontrollieren, ob auch Segelmaterial von beiden Segeln gefasst wurde, sollte man sichergehen, dass die Mitralklappe nun zwei Öffnungen besitzt und dass die Klappensegelanteile im Bereich der Clip-Arme immobilisiert sind und sicher aufliegen. Ist der Untersucher mit dem Ergebnis des Greifversuchs zufrieden, senkt er die Greifer ab und die Mitralsegel sind im Clip gefangen. Nun wird der Clip bis auf ca. $60^{\circ}$ geschlossen und die Verringerung der Mitralinsuffizienz dopplerechokardiographisch kontrolliert. Der Clip kann nun noch weiter geschlossen werden, bis die Mitralinsuffizienz akzeptabel verringert ist oder aber wieder geöffnet werden, um an einer geeigneteren Stelle positioniert zu werden. Der Untersucher sollte nicht mit übermäßigem Kraftaufwand versuchen, die Segel zu sehr zu spannen. Dies kann Probleme bei der Freisetzung des Clips verursachen sowie zu Verletzungen der Segel an den Stellen der Greifarme führen, die dann im ungünstigsten Fall zu der Lösung des Clips an diesem Segel führen könnten. Außerdem sollte der Operateur vor der Freisetzung des Clips den Gradienten über der Mitralklappe messen lassen, da bei einer Verringerung der Klappenöffnungsfläche auch immer die Gefahr einer Stenosierung besteht. Wenn der Untersucher von einer optimalen Position des Clips überzeugt ist, trennt er den Clip vom Clip-Einführsystem über Hebel am proximalen Ende des Clip-Einführsystems und setzt inn damit frei. Nun wird das Ergebnis nochmals echokardiographisch kontrolliert (vgl. Abb. 12) und es kann gegebenenfalls ein zweiter MitraClip® implantiert werden.

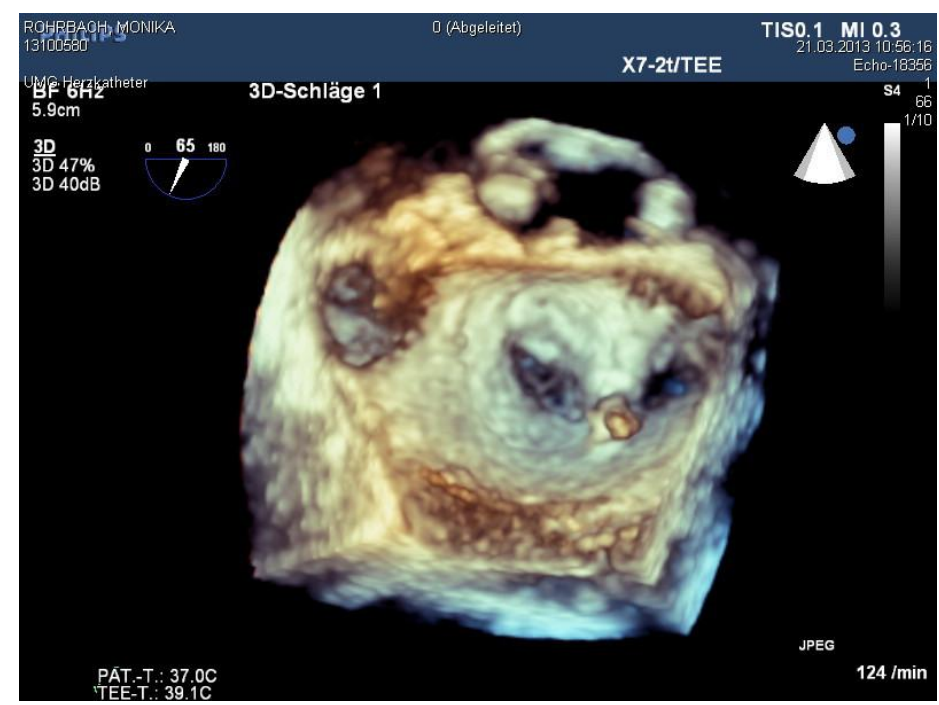

Abbildung 12: double-orifice der Mitralklappe nach Clip-Implantation 
Bei hämodynamisch relevanten Vorhofseptumdefekten kann an dieser Stelle noch die Implantation eines Vorhofseptum-Occluders diskutiert werden. Andernfalls werden das ClipEinführsystem sowie der steuerbare Führungskatheter unter Berücksichtigung der Gefahr der Luftembolie zurückgezogen und die Punktionsstelle in der Leiste verschlossen (evalve 2011; Feldman et al. 2005).

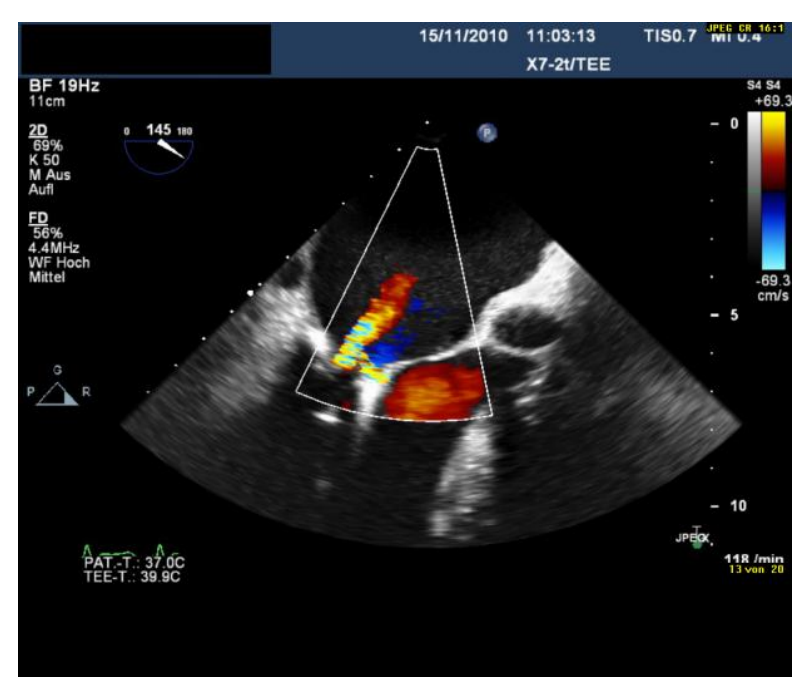

Abbildung 13

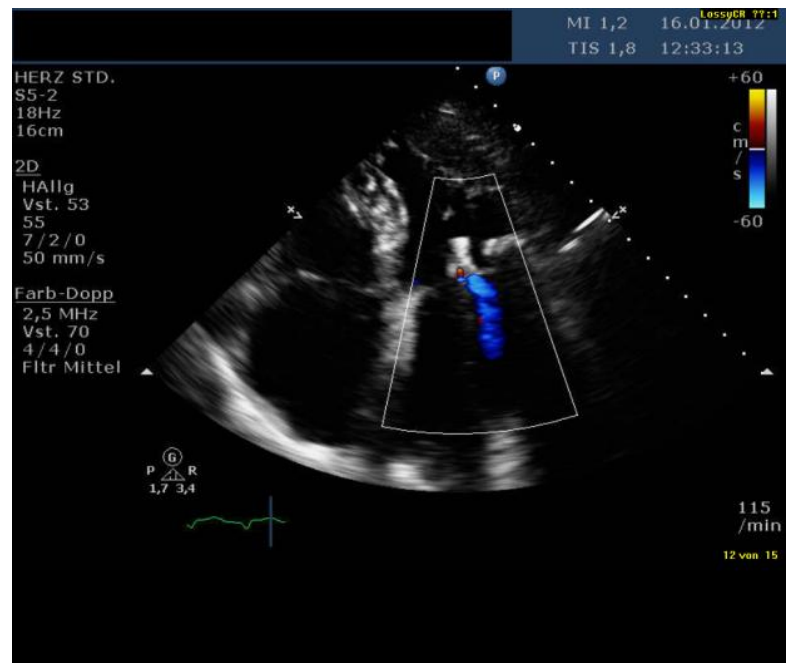

Abbildung 14

Ergebnis der perkutanen Mitralklappenreparatur postinterventionell (Abb.13) und nach einem Jahr (Abb.14)

\subsection{STATISTIK}

Die statistischen Auswertungen wurden mit Unterstützung der Abteilung Medizinische Statistik der Universitätsmedizin Göttingen durchgeführt. Für die Auswertung wurden die Programme Statistical Computing Software $R$ und graph pad prism version 4.0 benutzt. Kontinuierliche Variablen wurden (bei Abwesenheit einer Normalverteilung) als Mediane mit Quartilen ausgedrückt und mittels Mann-Whitney-Test sowie dem Wilcoxon-Vorzeichen-Rang-Test verglichen. Kategorische Variablen wurden als absolute Zahlen und Prozentangaben beschrieben und anhand des Chi-Quadrat-Tests miteinander verglichen. Ein $p$-Wert von < 0,05 wurde als signifikant angenommen. 
Überlebenszeitanalysen wurden vom Operationsdatum bis zum Ereignis (Hospitalisierung wegen Herzinsuffizienz oder Tod) mit Hilfe des $R$ package survival erstellt. Die Ergebnisse wurden mit Kaplan-Meier-Kurven visualisiert und unter Benutzung des log-rank-Testes auf Signifikanz überprüft. Bei kontinuierlichen Variablen wurde eine Aufteilung in zwei Gruppen mit Definition eines cut-off Wertes unternommen. Bei kategorischen Variablen oder bei multivariaten Modellen wurde das proportionale Hazardmodell verwendet. Analysen für Tod oder Rehospitalisierung wurden für Aufnahmeparameter, für Risikoberechnungsmodelle sowie den ausbleibenden Therapieerfolg erstellt. Mögliche Risikofaktoren, die in der univariaten Analyse eine Signifikanz zeigten, wurden anschließend in einer multivariaten Analyse mittels proportionalem Hazardmodell getestet. Den Einfluss der Risikoscores (z.B. log EuroSCORE, STS-Score oder das Vorhandenseins von Ausschlusskriterien der EVEREST-II-Studie) überprüften wir mit der Varianzanalyse ANOVA. Ein multivariates Cox-Modell (coxph) wurde benutzt, um verschiedene Parameter in unterschiedlichen Reihenfolgen zu überprüfen und so Abhängigkeiten der einzelnen Parameter untereinander aufzudecken 


\section{ERGEBNISSE}

\subsection{Basisdemographische Charakteristika Der Göttinger MITRACLIP®-KOHORTE}

Die ersten konsekutiven 150 Patienten, die im Herzzentrum der Universitätsmedizin Göttingen mittels MitraClip $\AA$ behandelt wurden, wiesen ein hohes Durchschnittsalter von $74.4 \pm 9.3$ Jahren sowie ein hohes geschätztes operatives Risiko bei einem logistischen EuroScore I von $28.6 \pm 17.9 \%$ sowie einem STS-Score von $10.5 \pm 8.7 \%$ auf. Bei einem Großteil der Patienten (66\%; $n=99$ ) war die Mitralinsuffizienz funktionell bedingt. Nahezu alle Patienten wiesen vor der Intervention eine hochgradige Symptomatik entsprechend der NYHA-Klassen III und IV auf $(87 \% ; n=130)$ und knapp die Hälfte der Patienten (45\%; $n=68)$ litt unter einer hochgradig eingeschränkten linksventrikulärer Funktion (LV-EF $\leq 30 \%$ ). Dies und die hohe Prävalenz von koronarer Herzkrankheit (62\%; $n=93$ ), von kardial voroperierten Patienten (36\%; $n=54)$, von Vorhofflimmern (66\%; $n=99$ ), von klinischen Zeichen der Rechtsherzinsuffizienz (45\%; $n=68)$ ebenso wie die hohe Prävalenz an nicht-kardialen Vorerkrankungen belegen das hohe Risiko der Patienten (vgl. Tabelle 1). 
Tabelle 1: Basisdemographische Charakteristika des Gesamtkollektivs ( $n=150)$

\section{Mittelwert \pm Standardabweichung}

Alter (Jahre)

$74.4 \pm 9.3$

Errechnetes peri-operatives Risiko

- Logistischer EuroScore I (\%)

$28.6 \pm 17.9$

- STS Score (\%)

$10.5 \pm 8.7$

$n$ (\%)

Weibliches Geschlecht

$53(35 \%)$

NYHA-Stadium III

$94(63 \%)$

NYHA-Stadium IV

$36(24 \%)$

Ätiologie der Mitralinsuffizienz:

- Primär (organisch)

$51(34 \%)$

- sekundär (funktionell)

$99(66 \%)$

LV-EF $\leq 30 \%$

$68(45 \%)$

Vorhandensein eines CRT-D

$38(25 \%)$

Koronare Herzerkrankung (KHK)

$93(62 \%)$

Vorausgegangene Herzoperation

$54(36 \%)$

Pulmonale Hypertonie (>50 mmHg)

$94(63 \%)$

Rechtsherzinsuffizienz (klin. Zeichen)

$68(45 \%)$

Vorhofflimmern

99 (66\%)

Periphere arterielle Verschlusskrankheit

$20(13 \%)$

Z.n. Apoplex

$12(8 \%)$

Chron. Niereninsuffizienz

- GFR $^{*}<30 \mathrm{~mL} / \mathrm{min} / 1,73 \mathrm{~m}^{2}$

$23(15 \%)$

- $\mathrm{GFR}^{*}<60 \mathrm{~mL} / \mathrm{min} / 1,73 \mathrm{~m}^{2}$

$101(67 \%)$

COPD

$33(22 \%)$

Diabetes mellitus

$47(31 \%)$

Katecholaminpflichtigkeit

$22(15 \%)$

Einschlusskriterien für EVEREST II erfüllt

$49(33 \%)$ 


\subsection{SICHERHEIT DER INTERVENTION UND PROZEDURALE ERGEBNISSE}

Die Prozedur konnte mit relativ großer Sicherheit durchgeführt werden. Kein Patient verstarb während des Eingriffes oder erlitt einen Myokardinfarkt. Ebenso war in keinem der Fälle eine unmittelbare chirurgische Korrektur oder der Gebrauch von Herzunterstützungssystemen notwendig.

Insgesamt wurden bei 23 der 150 Patienten (15\%) prozedurabhängige Komplikationen innerhalb des stationären Aufenthaltes nach Eingriff beobachtet (siehe Tabelle 3). Acht Personen verstarben intrahospital (innerhalb von 34 Tagen). Vier der Patienten erlitten aufgrund von Blutungen weitere Komplikationen wie Langzeitbeatmung mit konsekutiver Pneumonie, akutes Nierenversagen oder ischämischen Apoplex und verstarben letztendlich im septischen Schock (Tag 12, 24, 33, 34). Drei weitere Patienten starben ebenfalls an einer Sepsis, allerdings ohne zuvor eine Blutungskomplikation erlitten zu haben. Ein Patient starb an therapieresistentem Kammerflimmern (Tag 10).

Sieben Patienten mussten innerhalb von 30 Tagen aufgrund von manifester Herzinsuffizienzsymptomatik wiederum stationär behandelt werden. Eine nach den GUSTO-Kriterien (1993) als schwerwiegend definierte Blutung (intrazerebrale oder den Kreislauf beeinträchtigende, behandlungsnotwendige Blutung) erlitten sieben Patienten.

Eine weitere Komplikation war die Notwendigkeit der Verlängerung der Beatmungstherapie über 24 Stunden hinaus bei sechs Patienten.

Insgesamt 122 Patienten (85\%) konnten erfolgreich (Reduktion des Schweregrades der Mitralinsuffizienz auf $\leq 2$ ) mit MitraClip $\AA$ behandelt werden. Eine komplette Lösung eines freigesetzten Clips von beiden Segeln der Mitralklappe mit Embolisation wurde bei keinem der Patienten beobachtet. Jedoch wurden insgesamt vier partielle Cliplösungen registriert, bei denen der Clip jeweils die Bindung zu einem der Segel verlor. Zwei dieser Patienten konnten noch während der Intervention mit einem weiteren Clip versorgt und so erfolgreich behandelt werden. In einem Fall wurde im weiteren Verlauf die Mitralklappe chirurgisch ersetzt. Ein weiterer Patient verstarb, bevor er der schon geplanten chirurgischen Therapie zugeführt werden konnte (vgl. Tabelle 2). 
Tabelle 2. Periprozedurale Komplikationen im Gesamtkollektiv ( $n=150)$

$n(\%)$

Tod

$8(5.3 \%)$

Rehospitalisierung aufgrund von Herzinsuffizienz

$7(4.7 \%)$

Schwere Blutung (GUSTO) *

$7(4.7 \%)$

Myokardinfarkt

0

Apoplex (ischämisch/ hämorrhagisch)

$2(1.3 \%)$

Herzchirurgie

0

Herzunterstützungsystem

0

Perikardtamponade

$1(0.7 \%)$

Beatmungsdauer $>24 \mathrm{~h}$

$6(4.0 \%)$

Phlebothrombose

$2(1.3 \%)$

Dialysepflichtigkeit

$2(1.3 \%)$

Partielle Clip-Lösung

$4(2.6 \%)$

- intraprozedural

$3(2.0 \%)$

- postprozedural

$1(0.7 \%)$

Patienten mit Ereignis

$23(15 \%)$

*intrakranielle Blutung oder Blutung mit konsekutiver hämodynamischer Instabilität 


\subsection{6-MONATE-FOLLOW-UP}

Bei 140 der 150 behandelten Patienten lag die Intervention bereits $6( \pm 2)$ Monate zurück. Allerdings waren 20 Personen im Nachbeobachtungszeitraum verstorben und weitere 24 Patienten konnten entweder nur telefonisch befragt werden oder verweigerten ihre Teilnahme an einer Nachuntersuchung. Für die Analyse der Überlebenskurven und Hospitalisierungsrate war die Information zu diesen Patienten jedoch vorhanden und konnte in die Risikokalkulation eingehen. Ein komplettes Follow-Up mit vollständiger Echokardiographie absolvierten 96 der 150 Patienten. Für alle folgenden Analysen wurden nur Probanden mit vorhandenen Baseline und 6-Monats-Messungen berücksichtigt (gepaarte Analysen).

Bei 79,2\% der Patienten lag nach 6 Monaten ein stabiles Ergebnis der Prozedur mit einer residualen Mitralinsuffizienz des Schweregrades $\leq 2$ vor, wobei sich 73,7\% der Patienten zum Zeitpunkt der Untersuchung innerhalb der NYHA-Klassen I und II befanden (bei Aufnahme nur 13\%). Des Weiteren konnte bei den Patienten eine signifikante Steigerung der Lebensqualität beobachtet werden, die mit Hilfe des MLHFQ Testes gemessen wurde. Hier ergab sich eine Senkung des medianen Punktescores um 10 Punkte (37 auf 27 Punkte, $p<0,0001$; Abb. 15 B) im Vergleich zu der Baseline-Erhebung. Im ebenfalls zu beiden Zeitpunkten durchgeführten Sechs-Minuten-Gehtest erhöhte sich die mediane Gehstrecke von 290 Metern vor ClipImplantation auf 370 Meter nach sechs Monaten ( $p=0,0003$; Abb. 15 A). Außerdem konnte echokardiographisch ein reverses linksventrikuläres Remodeling anhand der Echokardiographieparameter beobachtet werden. So reduzierten sich sowohl der linksventrikuläre enddiastolische Diameter (LVEDD) (median; $59 \mathrm{~mm}$ auf $58 \mathrm{~mm}$; $\mathrm{p=0,003;} \mathrm{Abb.} 15 \mathrm{C}$ ) als auch das linksventrikuläre enddiastolische Volumen (LVEDV) (median; $164 \mathrm{ml}$ auf $141 \mathrm{ml}$; $\mathrm{p}=0,0001 ; 15 \mathrm{D})$. Weiterhin zeigte sich ein signifikanter Abfall der medianen NT-proBNPSpiegel im Vergleich zum Ausgangswert (2461 vs. 1986 pg/ml, $p=0,01$ ).

\subsection{2-MONATE -FOLLOW-UP}

Im Beobachtungszeitraum waren bei 94 Patienten bereits 12 Monate seit der jeweiligen ClipImplantation vergangen. Insgesamt 63 Patienten konnten wiederum zum Follow-Up einbestellt werden, da 20 Patienten verstorben waren und 11 Patienten nur einem telefonischen Gespräch zustimmten. 85,7\% der Patienten hatten weiterhin eine Mitralinsuffizienz des Schweregrades $\leq 2$, und $70,8 \%$ waren den NYHA-Klassen I und II zuzuordnen. Für alle folgenden statistischen Analysen wurden wieder nur Probanden mit vorhandenen Baselineund 12-Monats-Messungen berücksichtigt. Hier zeigte sich eine weitere Verbesserung der Lebensqualität, quantifiziert durch einen Abfall des medianen MLHFQ-Punktewerts von 43 auf 
$24(p=0,0001$; Abb. $15 \mathrm{~F})$, sowie eine signifikante Zunahme der Wegstrecke innerhalb des Sechs-Minuten-Gehtests von 315 Metern auf 400 Meter ( $p=0,02$; Abb. 15 E). Auch echokardiographisch konnten Veränderungen hinsichtlich der Dimensionen des linken Ventrikels beobachtet werden. Dabei verringerten sich der mediane LVEDD $(61,5 \mathrm{~mm}$ auf 59,5 mm; $\mathrm{p}=0,005$; Abb. $15 \mathrm{G}$ ) und das mediane LVEDV (199,5 ml auf $170 \mathrm{ml}$; $\mathrm{p}<0,0001$; Abb. $15 \mathrm{H}$ ) sowie das LVESV (median; $123 \mathrm{ml}$ auf $105 \mathrm{ml}$; $\mathrm{p}=0,004$ ). Bei der linksventrikulären Ejektionsfraktion und dem NT-proBNP gab es hingegen keine signifikanten Änderungen im Vergleich zum Ausgangswert.

A

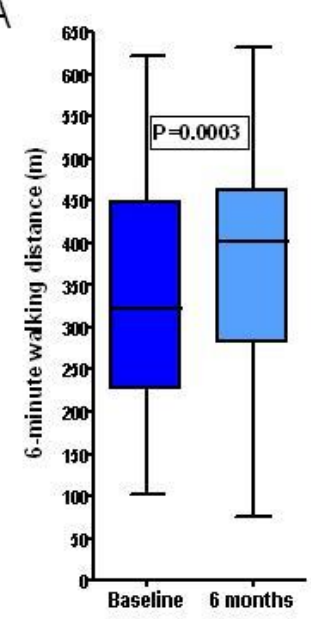

E

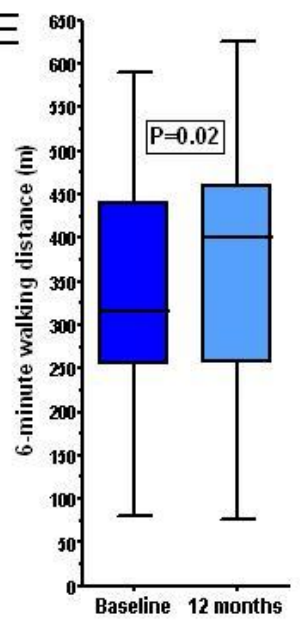

B

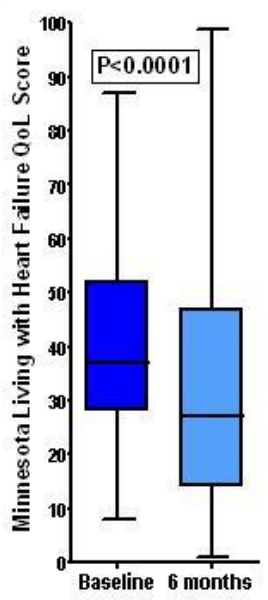

$\mathrm{F}$

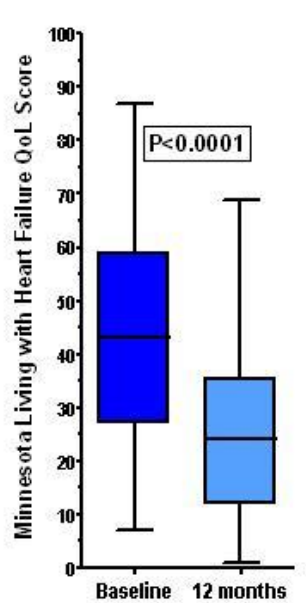

C

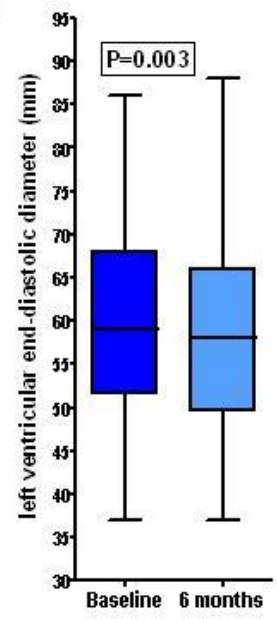

G

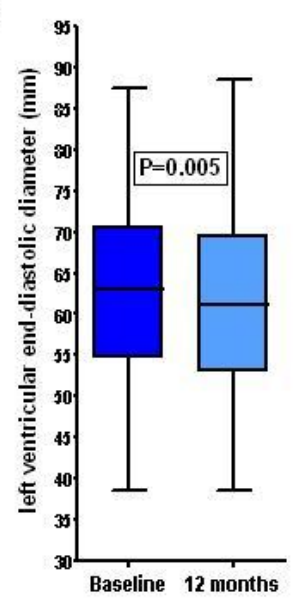

D

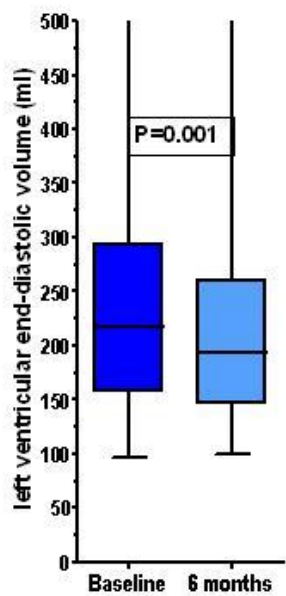

$\mathrm{H}$

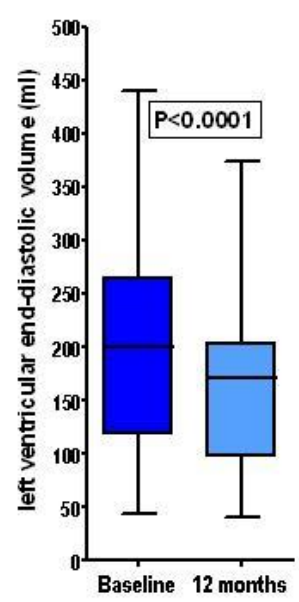

Abbildung 15: 6- und 12-Monatsdaten der Follow-Up-Untersuchungen 


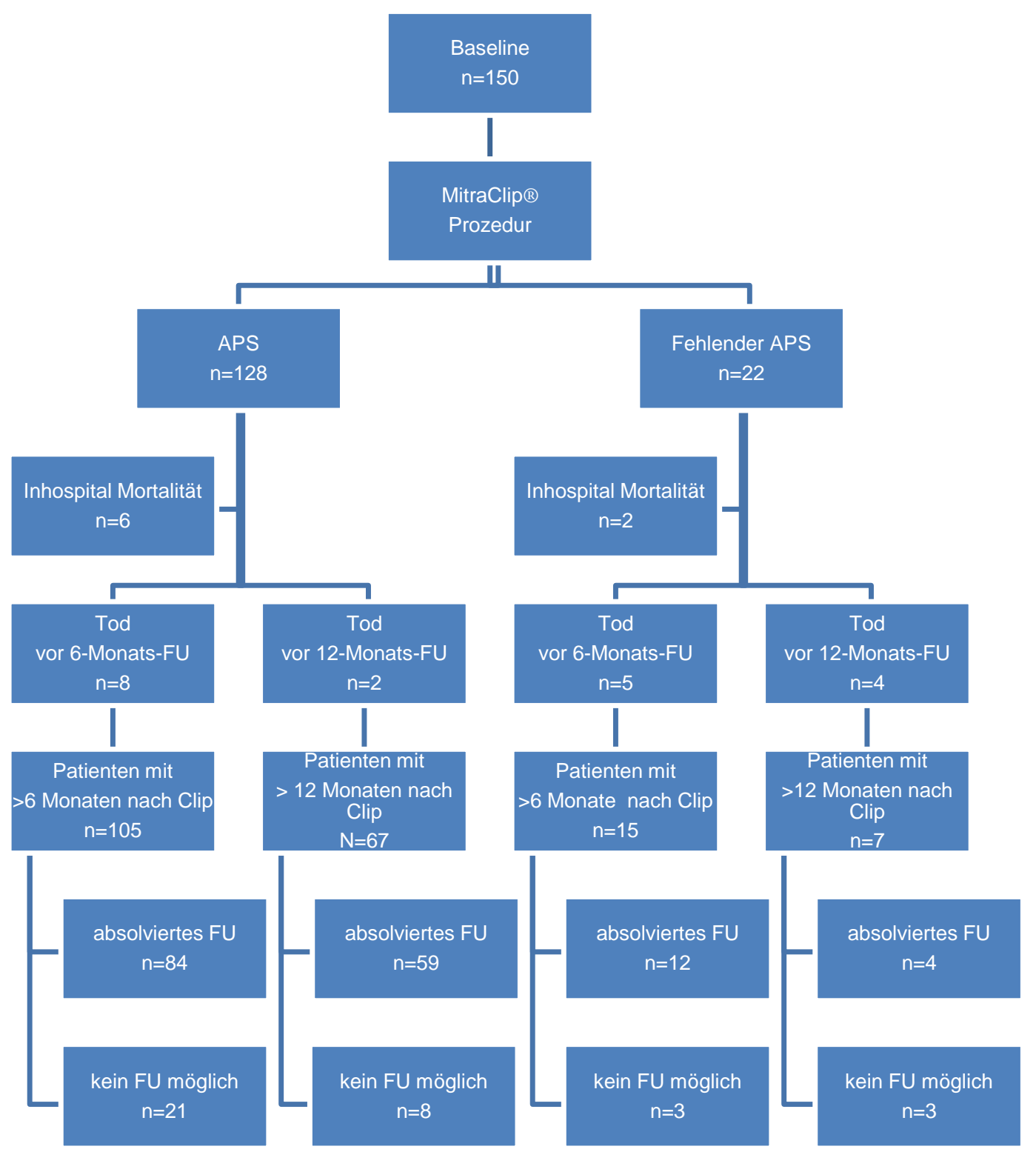

Abbildung 16: Studienprotokoll der Göttinger MitraClip®-Kohorte

Abbildung 16 zeigt die Übersicht über das Studienkollektiv der Göttinger MitraClip®-Patienten. Zunächst werden die Patienten in zwei Gruppen nach Behandlungsergebnis (Behandlungserfolg vs. Misserfolg) aufgeteilt. Außerdem werden die jeweiligen Beobachtungszeiträume (perioperativ, 6 und 12 Monate) aufgezeigt. 


\subsection{Kaplan-Meier-Analyse ÜBerLeben}

Die mediane Beobachtungsdauer der Patienten betrug 274 Tage. Die Überlebensrate (Kaplan-Meier-Analyse) lag nach 30 Tagen bei 96\%, nach 6 Monaten bei 85,1\% nach einem Jahr bei $78,9 \%$ und nach zwei Jahren bei $63 \%$. Insgesamt starben im gesamten Beobachtungszeitraum 39 Patienten (26\%). Die 8 intrahospitalen Todesfälle wurden bereits näher erläutert. Die Todesursachen der übrigen 31 Patienten waren: Kongestive Herz-insuffizienz und/ oder dokumentierte ventrikuläre Rhythmusstörungen ( $n=12)$, plötzlicher unerwarteter Tod $(n=6)$, peri-operative Komplikationen nach konventioneller Mitralklappenchirurgie $(n=2)$, akutes Nierenversagen $(n=3)$, Pneumonie $(n=3)$, septischer Schock infolge eines lleus $(n=1)$, intrakranielle Blutung $(n=1)$, erwarteter Tod in schlechtem Allgemeinzustand ohne weitere klassifizierbare Ursache $(n=2)$, und unbekannt $(n=1)$.

Mit Hilfe von Überlebenszeitanalysen wurden Baseline-Parameter, Risiko-Punktescores und das Vorhandensein eines Behandlungserfolges auf ihre Vorhersagekraft hinsichtlich des Überlebens der Patienten getestet. Dabei konnte in der univariaten Analyse nachgewiesen werden, dass Patienten, die zum Zeitpunkt der Aufnahme eine Dyspnoesymptomatik in Ruhe (NYHA-Stadium IV) oder klinische Zeichen einer Rechtsherzinsuffizienz zeigten, unter einer stark eingeschränkten Nierenfunktion (GFR<30 ml/min/1,73m²) litten, hohe Risikoscorewerte aufwiesen ( logistischer EuroScore I $\geq 20 \%$ oder STS Score $\geq 12 \%$ ) oder nicht erfolgreich mittels MitraClip $\AA$ behandelt werden konnten (persistierende Mitralinsuffizienz des Schweregrades >2), ein signifikant schlechteres Langzeitüberleben zeigten (vgl. Tabelle 3a). Auffällig war, dass andere für die konventionelle Herzchirurgie etablierte Risikofaktoren wie hohes Lebensalter, weibliches Geschlecht, ein vorausgegangener herzchirurgischer Eingriff, eine pAVK, eine LV-EF $<30 \%$ und eine COPD keine Vorhersagekraft hinsichtlich des Überlebens nach MitraClip® besaßen (vgl. Tabelle 3a). 
Tabelle 3a: Univariate Analyse möglicher Risikoprädiktoren

\section{Risikofaktor}

Lebensalter $\geq 70$ Jahre

Weibliches Geschlecht

\section{Ätiologie der Mitralinsuffizienz}

Primäre/ degenerative MI

Sekundäre/ funktionelle MI

\section{Kardiale Begleiterkrankungen}

DCM
ICM
LV-EF $<30 \%$
LVESD $\geq 45 \mathrm{~mm}$
LVESD $\geq 55 \mathrm{~mm}$
LVEDV $\geq 145 \mathrm{ml}$ (=median)
LVESV $\geq 81 \mathrm{ml}$ (=median)
MI Grad 4 vor Behandlung
NYHA IV
Katecholaminpflichtigkeit vor Intervention
Rechtsherzinsuffizienz (klin. Zeichen)
Trikuspidalklappeninsuffizienz
Pulmonale Hypertonie (PAPs $>50$ mmHg)
Vorhofflimmern
Koronare Herzerkrankung
Vorausgegangene PTCA
Vorausgegangene Herzoperation
Vorhandensein eines CRT-D

\section{Nicht-kardiale Begleiterkrankungen}

Vorausgegangener Apoplex

Periphere arterielle Verschlusskrankheit

GFR $<30 \mathrm{~mL} / \mathrm{min} / 1,73 \mathrm{~m}^{2}$

GFR $<60 \mathrm{~mL} / \mathrm{min} / 1,73 \mathrm{~m}^{2}$

COPD

Diabetes mellitus

\section{Risikoscores}

Log. EuroScore $I \geq 20 \%$

STS-Score $\geq 12 \%$

Ausschlusskriterien für EVEREST ॥

Akutes prozedurales Versagen

\section{Gesamtmortalität}

Hazard Ratio* P [logrank]

$1.07[0.5-2.3] \quad 0.85$

$1.40[0.7-2.7] \quad 0.30$

$\begin{array}{ll}0.92[0.5-1.8] & 0.80 \\ 0.96[0.5-1.8] & 0.90\end{array}$

$0.98[0.5-2.0] \quad 0.96$

$1.09[0.6-2.0] \quad 0.80$

$1.30[0.7-2.5] \quad 0.41$

$0.85[0.4-1.6] \quad 0.63$

$1.43[0.7-2.8] \quad 0.30$

$1.39[0.7-2.7] \quad 0.33$

$1.22[0.6-2.4] \quad 0.55$

$1.4[0.7-2.8] \quad 0.33$

$2.76[1.5-5.2] \quad \mathbf{0 . 0 0 1}^{*}$

$1.53[0.7-3.5] \quad 0.30$

$2.00[1.1-3.8] \quad \mathbf{0 . 0 3}^{*}$

$1.41[0.8-2.6] \quad 0.29$

$1.60[0.8-3.2] \quad 0.18$

$1.62[0.8-3.3] \quad 0.19$

$0.91[0.5-1.7] \quad 0.77$

$2.74[1.0-7.8] \quad 0.058$

$1.09[0.6-2.1] \quad 0.79$

$0.78[0.4-1.6] \quad 0.52$

$\begin{array}{ll}0.34[0.1-2.5] & 0.27 \\ 1.11[0.5-2.5] & 0.81 \\ \mathbf{2 . 3 3}[1.2-4.7] & 0.015^{\star} \\ 1.71[0.8-3.6] & 0.16 \\ 1.66[0.8-3.4] & 0.15 \\ 0.98[0.5-1.9] & 0.95\end{array}$

$2.25[1.0-4.9] \quad 0.035^{\star}$

$2.07[1.1-3.9] \quad \mathbf{0 . 0 2}$ *

$2.21[1.0-5.0] \quad 0.05$

$3.21[1.6-6.4] \quad<0.001^{*}$ 
Alle Faktoren, die in der univariaten Analyse eine statistische Signifikanz aufwiesen, wurden anschließend in der multivariaten Analyse mit dem proportionalen Hazard-Modell getestet. In diesem Test zeigten allein das Stadium NYHA IV $(p=0,02)$ sowie das akute prozedurale Versagen, definiert als persistierende Mitralinsuffizienz des Schweregrades $>2$ nach Prozedur, $(p=0,005)$ eine statistische Signifikanz.

Die herzchirurgischen Punktescores - wie der logistische EuroScore I und der STS-Score wurden mit Hilfe der Varianzanalyse ANOVA auf ihr Potenzial als Prädiktoren für die Prognose nach MitraClip®-Implantation überprüft. Hier konnte der EuroScore, wenn der STS-Score schon in das Modell eingefügt wurde, keine weiteren Informationen liefern. Dagegen zeigte der STS-Score, auch wenn schon alle anderen Parameter in das Modell eingesetzt worden waren, immer noch einen zusätzlichen Einfluss. In der ANOVA-Analyse verlor die Herzinsuffizienz im Stadium NYHA IV ihren prädiktiven Werte bei Vorhandensein des STSScores im Modell (vgl. Tabelle 3b).

Tab. 3b: Multivariate Analyse univariat signifikanter Risikofaktoren

\section{Gesamtmortalität}

Hazard Ratio*

$\mathbf{P}[\mathrm{coxph}]$

\section{Begleiterkrankung}

NYHA IV

$2.12[1.1-4.1]$

$0.02^{*}$

Rechtsherzinsuffizienz

GFR $<30 \mathrm{~mL} / \mathrm{min} / 1,73 \mathrm{~m}^{2}$

$1.79[0.9-3.5]$

0.08

$1.71[0.8-3.5]$

0.15

\section{Risikoscore}

Log. EuroScore I $\geq 20 \%$

STS-Score $\geq 12 \%$

Akutes prozedurales Versagen

$$
\text { / (ANOVA) }
$$

/ (ANOVA)

$<0.0001^{*}$

$2.73[1.3-5.5]$ $0.005^{\star}$

Zusammenfassend konnten das akute prozedurale Versagen sowie ein STS-Score $\geq 12$ als hochsignifikante unabhängige Prädiktoren der Gesamtmortalität im Follow-Up identifiziert werden. Abbildung 17 zeigt Kaplan-Meier-Kurven, die den Einfluss des akuten Prozedurversagens auf das Langzeitüberleben verdeutlichen. 


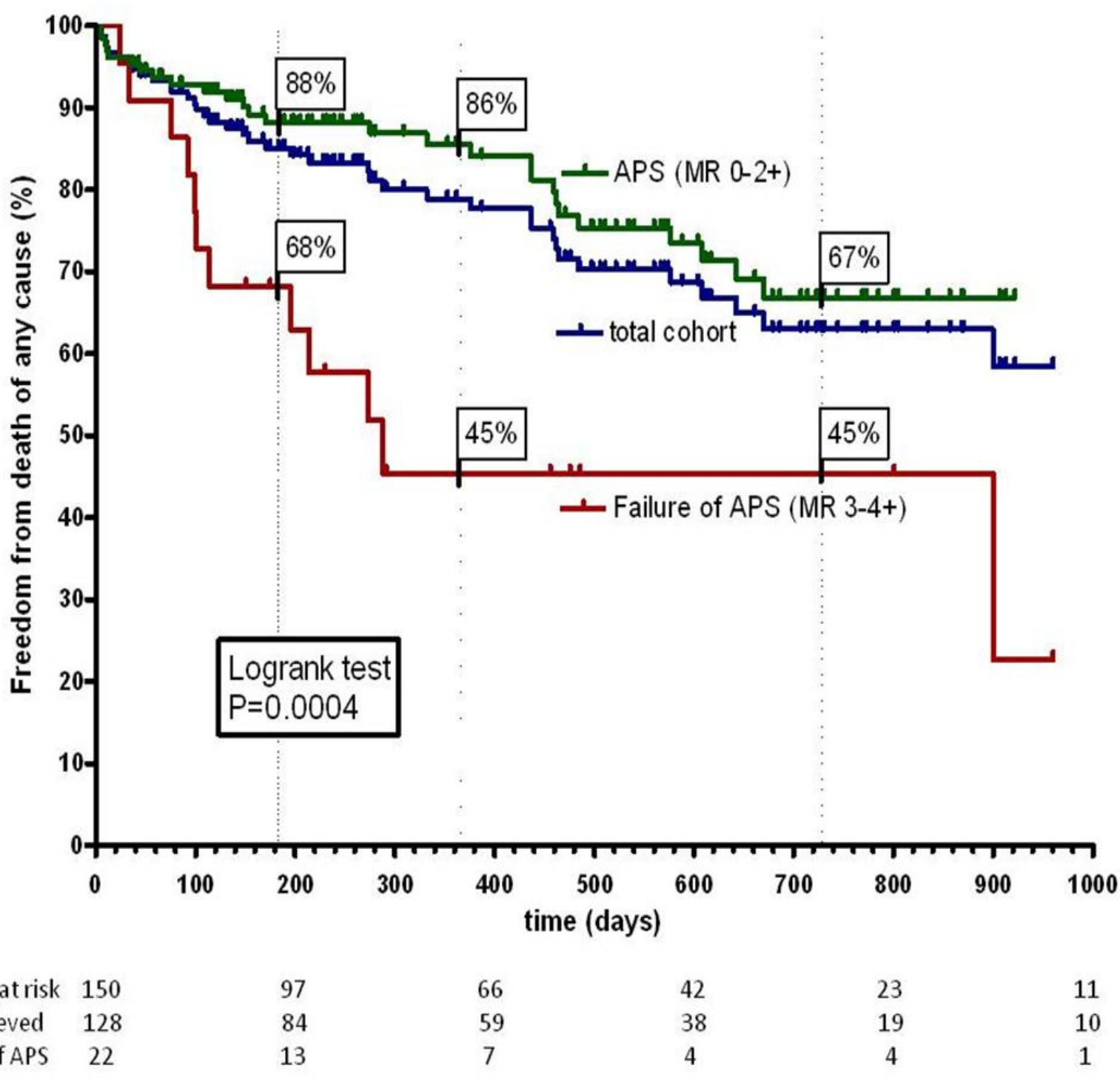

Abbildung 17: Kaplan-Meier-Kurven der Gesamtmortalität im Follow-up in Abhängigkeit vom Erreichen eines akuten prozeduralen Erfolges (APS)

\subsection{Kaplan-Meier-Analyse Hospitalisierung Wegen Herzinsuffizienz}

Analog zu den Auswertungen zum Überleben der Patienten wurden Prädiktoren für die erneute herzinsuffizienzbedingte Hospitalisierung nach MitraClip®-Implantation identifiziert. Bei der univariaten Analyse waren eine LV-EF $<30 \%$, ein LV-EDD $\geq 55 \mathrm{~mm}$, das Vorhandensein von Ausschlusskriterien für EVEREST II (Mauri et al. 2010) sowie eine erfolglose Behandlung mit MitraClip® signifikante Prädiktoren für die erneute Hospitalisierung aufgrund von Herzinsuffizienz nach Intervention (vgl. Tabelle 4a). 


\begin{tabular}{|c|c|c|}
\hline \multicolumn{3}{|c|}{ Tabelle 4a Univariate Analyse möglicher Prädiktoren der Rehospitalisierung } \\
\hline Risikofaktor & Hazard Ratio & $\mathbf{P}$ [logrank] \\
\hline Alter $\geq 70$ Jahre & $0.73[0.4-1.3]$ & 0.298 \\
\hline Weibliches Geschlecht & $1.13[0.6-2.0]$ & 0.661 \\
\hline \multicolumn{3}{|l|}{ Ätiologie der MI } \\
\hline Primäre / Degenerative MI & $0.63[0.3-1.2]$ & 0.127 \\
\hline Sekundäre / Funktionale MI & $1.37[0.8-2.5]$ & 0.291 \\
\hline \multicolumn{3}{|l|}{ Kardiale Begleiterkrankungen } \\
\hline $\mathrm{DCM}$ & $1.74[1.0-3.1]$ & 0.051 \\
\hline ICM & $0.82[0.5-1.5]$ & 0.494 \\
\hline LV-EF $<30 \%$ & $1.99[1.1-3.5]$ & $0.013^{\star}$ \\
\hline LVESD $\geq 45 \mathrm{~mm}$ & $1.17[0.7-2.0]$ & 0.584 \\
\hline LVESD $\geq 55 \mathrm{~mm}$ & $1.9[1.1-3.4]$ & $0.0258^{\star}$ \\
\hline LVEDV $\geq 145 \mathrm{ml}$ (=median) & $0.84[0.5-1.5]$ & 0.527 \\
\hline LVESV $\geq 81 \mathrm{ml}$ (=median) & $0.74[0.4-1.3]$ & 0.273 \\
\hline MI Grad 4 vor Behandlung & $0.69[0.4-1.2]$ & 0.188 \\
\hline NYHA IV & $1.53[0.8-2.9]$ & 0.181 \\
\hline Katecholaminpflichtigkeit vor Intervention & $1.42[0.7-3.0]$ & 0.367 \\
\hline Rechtsherzinsuffizienz (klin. Zeichen) & $1.00[0.6-1.7]$ & 1.000 \\
\hline Trikuspidalinsuffizienz & $0.77[0.4-1.4]$ & 0.357 \\
\hline Pulmonale Hypertonie (PAPs $>50 \mathrm{mmHg}$ ) & $0.99[0.6-1.7]$ & 0.969 \\
\hline Vorhofflimmern & $1.31[0.7-2.4]$ & 0.375 \\
\hline Koronare Herzkrankheit & $1.02[0.6-1.8]$ & 0.958 \\
\hline Vorausgegangene PTCA & $2.11[0.8-5.9]$ & 0.144 \\
\hline Vorheriger Herzchirurgischer Eingriff & $0.93[0.5-1.7]$ & 0.799 \\
\hline Vorhandensein eines CRT-D & $1.41[0.8-2.5]$ & 0.253 \\
\hline \multicolumn{3}{|l|}{ Nicht-kardiale Begleiterkrankungen } \\
\hline Vorausgegangener Apoplex & $0.73[0.2-2.3]$ & 0.591 \\
\hline Periphere arterielle Verschlusskrankheit & $0.74[0.3-1.7]$ & 0.476 \\
\hline GFR $<30 \mathrm{~mL} / \mathrm{min} / 1,73 \mathrm{~m}^{2}$ & $0.64[0.3-1.6]$ & 0.339 \\
\hline GFR $<60 \mathrm{~mL} / \mathrm{min} / 1,73 \mathrm{~m}^{2}$ & $1.41[0.8-2.6]$ & 0.275 \\
\hline COPD & $0.85[0.4-1.7]$ & 0.656 \\
\hline Diabetes mellitus & $1.19[0.7-2.1]$ & 0.556 \\
\hline \multicolumn{3}{|l|}{ Risiko Scores } \\
\hline Log. EuroScore I $\geq 20 \%$ & $1.07[0.6-1.9]$ & 0.82 \\
\hline STS-Score $\geq 12 \%$ & $1.58[0.9-2.8]$ & 0.106 \\
\hline Ausschlusskriterien für EVEREST II & $1.98[1.0-3.8]$ & $0.035^{\star}$ \\
\hline Akutes prozedurales Versagen & $3.20[1.7-6.1]$ & $<0.001^{*}$ \\
\hline
\end{tabular}


Auch hier wurden die univariat signifikanten Parameter mittels multivariater Analyse überprüft. Das Fehlen des akuten Prozedurerfolgs war der einzige multivariat signifikante Parameter für erneute Hospitalisierung aufgrund von Herzinsuffizienz. Dieser Zusammenhang wird in Abbildung 18 mit Hilfe von Kaplan-Meier-Kurven aufgezeigt. Alle anderen univariat signifikant getesteten Parameter verloren ihre Signifikanz in der multivariaten Analyse (vgl. Tabelle 4b).

Tab. 4b: Multivariate Analyse der herzinsuffizienzbedingten Rehospitalisierung

Hazard Ratio*

$2.18[0.9-5.2]$

0.08

LV-EF $<30 \%$

LVESD $\geq 55 \mathrm{~mm}$

$0.99[0.96-1.0]$

\section{Risikoscores}

Ausschlusskriterien für EVEREST II / (ANOVA)

Akutes prozedurales Versagen

$2.69[1.4-5.3]$

$0.004^{*}$ 


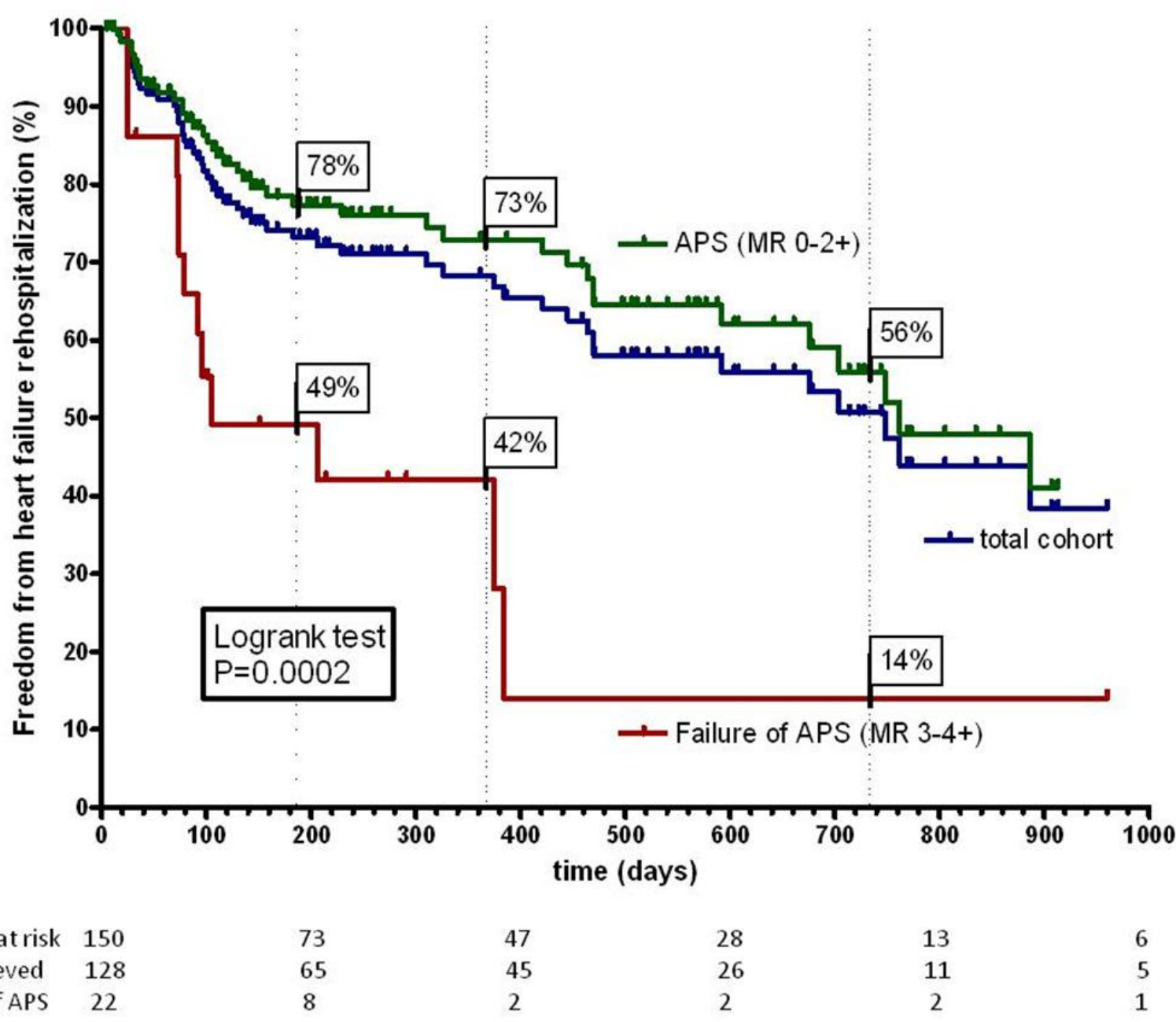

Abbildung 18: Kaplan-Meier-Kurven der herzinsuffizienzbedingten erneuten Hospitalisierung in Abhängigkeit vom Erreichen eines akuten prozeduralen Erfolges (APS)

\subsection{HeRZINSUfFIZIENZBEDINGTE HOSPITALISIERUNGSRATE VOR UND NACH INTERVENTION}

Wir verglichen weiterhin die Hospitalisierungsrate im Zeitraum von einem Jahr vor der Intervention mit dem Jahr nach MitraClipß-Implantation bei allen Patienten, die lebend aus dem Krankenhaus entlassen worden waren und deren Operation bereits mindestens ein Jahr zurücklag. Hier konnte gezeigt werden, dass bei Patienten mit Prozedurerfolg $(n=74)$ die Hospitalisierungsrate nach Clip-Implantation signifikant abnahm $(p=0,02)$. So betrug die Hospitalisierungsrate im Jahr vor Clip-Implantation 0,81 $\pm 0,95$, nach erfolgreicher Implantation hingegen $0,47 \pm 0,83$ pro Jahr. Konnte die Prozedur jedoch nicht erfolgreich durchgeführt werden (persistierende Mitralinsuffizienz $>2^{\circ}$ ), so war auch kein Unterschied in 
der Hospitalisierungsrate erkennbar ( $n=15 ; 1,53 \pm 2,07$ vs. $1,53 \pm 2,77 ; p=0,9)$ (vgl. Abbildung 19).

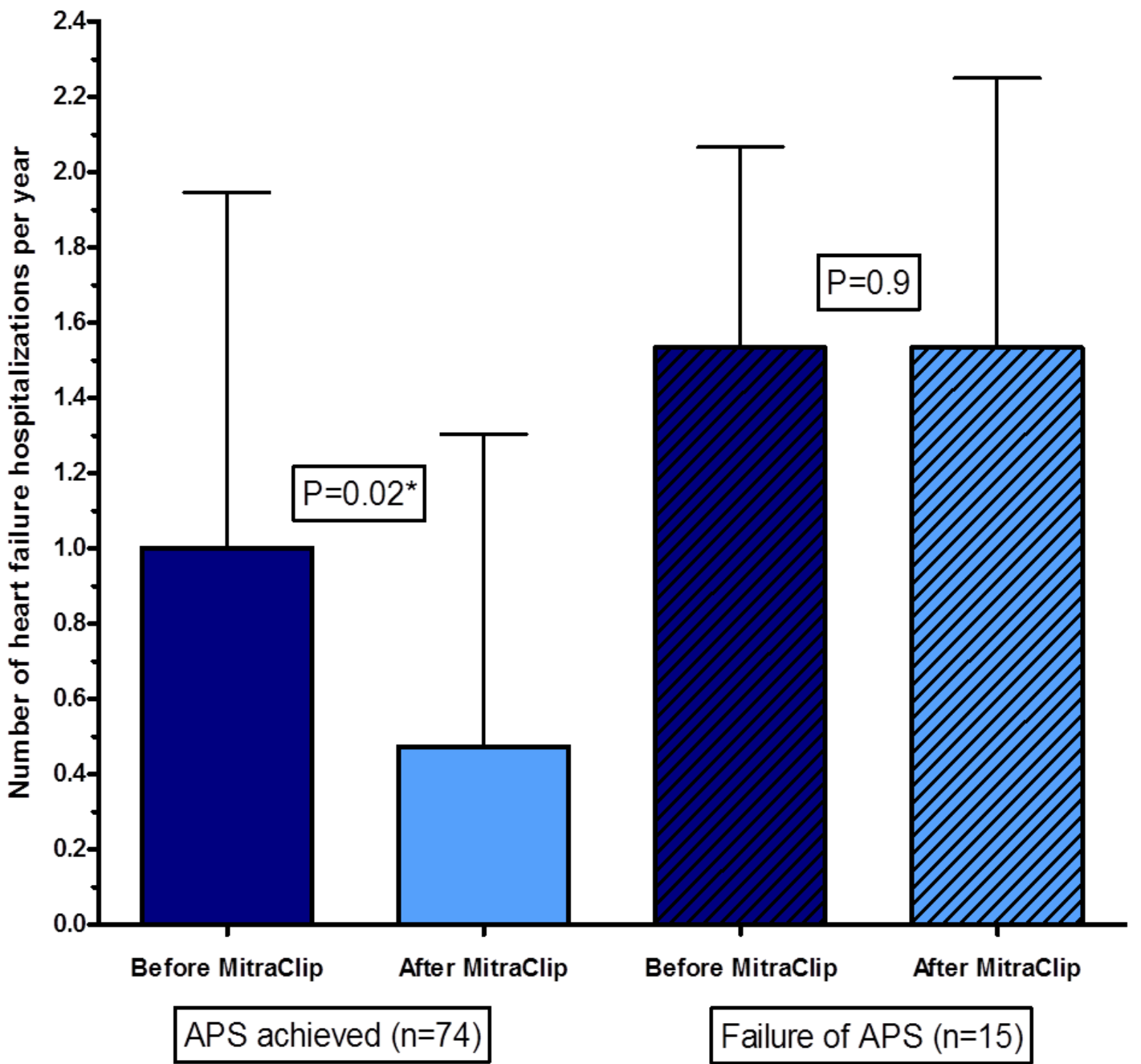

Abbildung 19: Anzahl herzinsuffizienzbedingter Hospitalisationen innerhalb eines Jahres vor und nach MitraClip®-Implantation

\subsection{PrädikToREn des AKUTEN PROZEdURALen VersagenS}

Um mögliche Prädiktoren des akuten Prozedurversagens zu ermitteln, verglichen wir basisdemographische Daten, echokardiographische Parameter und peri-interventionelle Messungen bei Patienten mit oder ohne akutem prozeduralem Erfolg.

Bei Untersuchung der basisdemographischen Daten waren zwischen den beiden oben genannten Patientengruppen lediglich zwei Merkmale signifikant unterschiedlich. Die späteren Therapieversager litten bedeutend häufiger unter einer pulmonalen Hypertonie (PAP syst. $>50 \mathrm{mmHg}$ ) und erfüllten signifikant seltener die EVEREST II-Einschlusskriterien (Mauri et al. 2010) $(37 \%$ vs. $9 \%, p=0,01)$. Keine Unterschiede gab es hingegen hinsichtlich der Ätiologie 
der Mitralinsuffizienz oder des per log. EuroSCORE I oder STS Score geschätzten perioperativen Risikos (vgl. Tabelle 5).

\section{Tabelle 5. Basisdemographische Charakteristika im Vergleich}

APS $^{*}(n=128) \quad$ kein APS $^{*}(n=22) \quad P$

\section{Mittelwert \pm SD}

Alter in Jahren

$74.7 \pm 9.3$

$72.5 \pm 9.5$

0.32

Errechnetes chirurgisches Risiko

- Logistischer EuroScore, \%

$28.2 \pm 17.3$

$30.8 \pm 21.7$

0.94

- STS Score, \%

$10.3 \pm 8.0$

$11.4 \pm 12.2$

0.56

$n$ (\%)

Weibliches Geschlecht

$46(36 \%)$

$7(32 \%)$

0.71

NYHA-Klasse III

$82(64 \%)$

$12(55 \%)$

0.39

NYHA-Klasse IV

$28(22 \%)$

$8(36 \%)$

0.14

Mechanismus der Mitralinsuffizienz

Primär
Sekundär
LV-EF $\leq 30 \%$
CRT-D

$46(36 \%)$

$5(23 \%)$

0.23

$82(64 \%)$

$17(77 \%)$

0.23

$55(43 \%)$

$13(59 \%)$

0.16

$31(24 \%)$

$7(32 \%)$

0.45

Koronare Herzkrankheit

$80(63 \%)$

$13(59 \%)$

0.76

Vorheriger herzchirurg. Eingriff

$44(34 \%)$

$10(45 \%)$

0.32

Pulm. Hypertension (>50 mmHg)

$76(59 \%)$

$18(82 \%)$

$0.04^{*}$

Rechtsherzinsuffizienz (klinisch)

$59(46 \%)$

$9(41 \%)$

0.65

Vorhofflimmern

$84(66 \%)$

$15(68 \%)$

0.97

pAVK

$15(12 \%)$

$5(23 \%)$

0.16

Vorheriger Schlaganfall

$12(9 \%)$

0

0.13

Chronische Niereninsuffizienz

- $\mathrm{GFR}^{*}<30 \mathrm{~mL} / \mathrm{min} / 1,73 \mathrm{~m}^{2}$

- $\mathrm{GFR}^{*}<60 \mathrm{~mL} / \mathrm{min} / 1,73 \mathrm{~m}^{2}$

$18(14 \%)$

$5(23 \%)$

0.30

$83(65 \%)$

$18(82 \%)$

0.12

COPD

$30(23 \%)$

$3(14 \%)$

0.31

Diabetes mellitus

$43(34 \%)$

$4(18 \%)$

0.15

Präop. Katecholaminpflichtigkeit

$19(15 \%)$

$3(14 \%)$

0.40

Eignung für EVEREST II

$47(37 \%)$

$2(9 \%)$

$0.01^{*}$ 
Weiterhin waren zwischen diesen beiden Patientengruppen Unterschiede in Bezug auf die echokardiographischen Parameter bei Aufnahme festzustellen. Die Messungen ergaben in der Patientengruppe ohne späteren Prozedurerfolg signifikant höhere end-diastolische Diameter und Volumina des linken Ventrikels. Ebenso waren die Mitralinsuffizienzen der Patienten ohne Behandlungserfolg höhergradiger (vgl. Tabelle 6).

\section{Tabelle 6. Präinterventionelle echokardiographische Messungen}

\begin{tabular}{|c|c|c|c|}
\hline & $\begin{array}{l}\text { APS }^{*} \\
(\mathrm{~N}=128)\end{array}$ & $\begin{array}{l}\text { Kein APS } \\
(\mathrm{N}=22)\end{array}$ & $\mathbf{P}$ \\
\hline \multicolumn{4}{|l|}{$n(\%)$} \\
\hline \multicolumn{4}{|c|}{ Mechanismus der Mitralinsuffizienz } \\
\hline Primär & $46(36 \%)$ & $5(23 \%)$ & 0.23 \\
\hline Sekundär & $82(64 \%)$ & $17(77 \%)$ & 0.23 \\
\hline - ICM & $53(64.6 \%)$ & $9(52.9 \%)$ & 0.36 \\
\hline - DCM & $29(35.45)$ & $8(47.1 \%)$ & 0.36 \\
\hline \multicolumn{4}{|c|}{ Schweregrad der Mitralinsuffizienz } \\
\hline Grad 2+ & $2(1.6 \%)$ & 0 & 0.56 \\
\hline Grad 3+ & $51(39.8 \%)$ & $3(13.6 \%)$ & $0.02^{*}$ \\
\hline Grad 4+ & $75(58.6 \%)$ & $19(86.4 \%)$ & $0.01^{*}$ \\
\hline PAsP $>50 \mathrm{mmHg}$ & $76(59 \%)$ & $18(82 \%)$ & $0.04^{\star}$ \\
\hline \multicolumn{4}{|c|}{ Median (25.-75. Perzentile) } \\
\hline LV-EF, \% & $36.5(25-51.5)$ & $30(18-47.5)$ & 0.12 \\
\hline LVEDD, mm & $58(50-67)$ & $65(56-73.5)$ & $0.037^{\star}$ \\
\hline LVEDS, mm & $44.5(37-56)$ & $54(37-61)$ & 0.22 \\
\hline LVEDV, ml & $144(92-221)$ & $191(135-257)$ & $0.038^{\star}$ \\
\hline LVESV, ml & $80(39-144)$ & $115(69-211)$ & $0.047^{\star}$ \\
\hline
\end{tabular}

Weiterhin wurden die peri-interventionellen Komplikationen innerhalb der ersten 30 Tage nach Intervention (bzw. bis zur Entlassung, falls diese erst nach mehr als 30 Tagen erfolgte) zwischen den beiden Patientengruppen mit und ohne Prozedurerfolg verglichen. Hier ergab sich kein Unterschied hinsichtlich der peri-operativen Mortalität. Allerdings erlitten Patienten ohne akuten Behandlungserfolg post-interventionell häufiger eine schwere Blutung (GUSTOKriterien) $(3,1 \%, n=4$ vs. $13,6 \%, n=3 ; p=0,03)$ und mussten öfter einer verlängerten Beatmungstherapie (>24h) zugeführt werden $(2,3 \% ; n=3$ vs. $13,6 \% ; n=3 ; p=0,01)$. Auch frühe 
herzinsuffizienzbedingte Rehospitalisierungen waren in der Gruppe der Therapieversager signifikant häufiger $(3,1 \%, n=4$ vs. $13,6 \%, n=3 ; p=0,03)$ (vgl. Tabelle 7$)$.

Tabelle 7. Peri-interventionelle Komplikationen (innerhalb von 30 Tagen bzw. bis zur Entlassung)

\section{APS $^{*}(n=128) \quad$ Kein APS $^{*}(n=22) \quad P$}

$n(\%)$

Tod

$6(4.7 \%)$

$2(9.1 \%)$

0.40

frühe Herzinsuff.-Rehospitalisierung

$4(3.1 \%)$

$3(13.6 \%)$

$0.03^{*}$

Schwere Blutung (GUSTO)

$4(3.1 \%)$

$3(13.6 \%)$

$0.03^{*}$

Myokardinfarkt

0

Apoplex (ischämisch/ hämorrhagisch)

$1(0.8 \%)$

0

Herzchirurgie

0

$1(4.5 \%)$

0.16

Herzunterstützungsystem

0

Perikardtamponade

$1(0.8 \%)$

0

0

/

Beatmungsdauer $>24 \mathrm{~h}$

$3(2.3 \%)$

0

0.68

Phlebothrombose

$2(1.6 \%)$

$3(13.6 \%)$

$0.01^{*}$

Dialysepflichtigkeit

$2(1.6 \%)$

0

0.56

Partielle Clip Lösung

$2(1.6 \%)$

0

0.56

- intraprozedural

$2(1.6 \%)$

$2(9.1 \%)$

$0.04^{*}$

- postprozedural

0

$1(4.5 \%)$

0.3

Patienten mit Ereignis

$15(12 \%)$

$1(4.5 \%)$

$0.02^{*}$

$8(36 \%)$

$0.003^{\star}$ 


\section{DISKUSSION}

Im Vergleich zu den Patienten der aktuell wichtigsten MitraClip®-Studie, der EVEREST-IIKohorte, waren die Göttinger MitraClip®-Patienten älter (67 \pm 13 vs. $74 \pm 9$ Jahre), symptomatischer (Patienten in NYHA III+IV: $50 \%$ vs. $87 \%$ ) und litten unter deutlich mehr Begleiterkrankungen (erkennbar zum Beispiel am deutlich höheren STS Score: $5 \pm 4 \%$ vs. $10 \pm 9 \%)$. Außerdem war die Ätiologie der Mitralinsuffizienz in diesem Kollektiv anders verteilt: Während im EVEREST-II-Kollektiv die primäre (degenerative) Mitralinsuffizienz vorherrschend war $(73 \%$ der Patienten), waren die Mitralinsuffizienzen der Göttinger Patienten vornehmlich sekundären (funktionellen) Ursprungs (66\% der Patienten). In den europäischen Registern (ACCESS-EU-Register, deutsches Mitralklappenregister) entsprachen die Verteilungen der Mitralinsuffizienz-Ätiologien und der basisdemographischen Patientencharakteristika dem Göttinger Kollektiv. Dies ist wichtig, um einerseits die im Vergleich mit den EVEREST-Daten höheren Mortalitätsraten in unserer Kohorte zu verstehen, andererseits zeigt es aber auch, dass eine MitraClip®-Implantation bei einem hohen Prozentsatz kardiologischer Hochrisikopatienten sicher und erfolgreich durchführbar ist (85\% unserer Patienten hatten einen akuten Prozedurerfolg). Diese Tatsache bestätigt die Ergebnisse, die in der Vergangenheit auch schon an anderen Zentren (Pleger et al. 2011) und in den Registerstudien (Maisano et al. 2013; Schillinger et al. 2013) gezeigt werden konnten. In der vorliegenden Arbeit konnten nun aber zum ersten Mal Prädiktoren für Mortalität und herzinsuffizienzbedingte Rehospitalisierung in einem relativ großen Zentrumskollektiv ermittelt werden.

Eine symptomatische Herzinsuffizienz im Stadium NYHA IV vor der Prozedur, ein STS Score von $\geq 12 \%$ sowie ein akutes prozedurales Versagen (persistierende Mitralinsuffizienz $>2+$ ) konnten als signifikante Prädiktoren einer erhöhten Mortalität nach MitraClip®-Implantation in den multivariaten Analysen identifiziert werden. Bei Patienten, die diese Merkmale bei Aufnahme aufweisen, sollten das erhöhte Risiko und die zu erwartenden Erfolgsaussichten sorgfältig diskutiert werden. Das akute prozedurale Versagen war ebenfalls ein Prädiktor der herzinsuffizienzbedingten Rehospitalisierung nach MitraClip®-Implantation. Daher sollten bei den erfolglos behandelten Patienten zum einen eine aufmerksame Nachbeobachtung mit engen Untersuchungsintervallen durchgeführt werden, zum anderen sollten hier aufgrund der schlechten Prognose dieser Patienten zeitnah weitere Möglichkeiten der Intervention diskutiert werden. Zum Beispiel sind hier ein erneuter Versuch der Clip-Implantation oder ein herzchirurgischer Eingriff zu nennen, falls das operative Risiko noch vertretbar erscheint. In der Herzchirurgie etablierte Risikofaktoren wie vorherige herzchirurgische Eingriffe, eine koronare Herzkrankheit oder periphere Gefäßkrankheit zeigten dagegen keinen Einfluss auf 
Mortalität oder Rehospitalisierung nach MitraClipß und sollten daher nicht zur Ablehnung des Patienten für eine Clip-Implantation führen.

Vahanian et.al (Vahanian et al. 2012) beschreiben in den aktuellen Leitlinien noch weitere Prädiktoren für das Outcome nach chirurgischer Therapie der primären Mitralinsuffizienz. Hier sind das fortgeschrittene Lebensalter und das Vorhandensein von Vorhofflimmern oder eines pulmonalen Hypertonus mit einer schlechteren Prognose assoziiert. In der Göttinger MitraClip ${ }^{\circledR}$-Kohorte hatten diese Faktoren keinen Einfluss auf die Endpunkte Mortalität und Rehospitalisierung. In einer anderen Arbeit wurde ein linksventrikulärer endsystolischer Diameter $\geq 40 \mathrm{~mm}$ (LVESD) als unabhängiger Risikofaktor für Mortalität bei primärer Mitralinsuffizienz sowohl bei medikamentöser als auch bei chirurgischer Therapie identifiziert (Tribouilloy et al. 2009). Bei der sekundären Mitralinsuffizienz wurde ein LVEDD > 65mm als Prädiktor einer schlechten Prognose nach Annuloplastie ermittelt (Ciarka et al. 2010). Diese Parameter zeigten wie alle anderen Echoparameter in unserem Kollektiv keine Signifikanz hinsichtlich der Mortalität. Lediglich in der univariaten Analyse waren die LV-EF $<30 \%$ und der LVESD > 55mm signifikante Prädiktoren der postprozeduralen herzinsuffizienzbedingten Hospitalisierung.

Es gibt bisher zwei veröffentlichte Untersuchungen zu Outcome-Prädiktoren nach MitraClip®-Implantation. Beide kommen aus dem Rostocker Zentrum und umfassen eine Kohorte von 85 Patienten. Paranskaya et al. (Paranskaya et al. 2012a; Paranskaya et al. 2012b) zeigten an diesem Kollektiv Risikofaktoren für einen zusammengefassten Endpunkt von Tod, Rehospitalisierung aufgrund kardialer Ursache, Re-Intervention, zerebro-vaskuläre Komplikationen und kardiale Ereignisse. Dabei zeigten die Autoren, dass eine residuale Mitralinsuffizienz (jedes Schweregrades) die Inzidenz des kombinierten Endpunktes signifikant erhöht. Im Gegensatz dazu zeigte sich im Göttinger Kollektiv nur ein signifikant schlechteres Outcome in der Gruppe der Patienten mit persistierender Mitralinsuffizienz des Schweregrades 3 oder 4 (definiert als akutes prozedurales Versagen), während das Outcome bei persistierender Mitralinsuffizienz des Schweregrades 2, 1 oder 0 nicht signifikant unterschiedlich war.

Mit einer Anzahl von 150 Patienten gehört das Göttinger MitraClip®-Kollektiv im weltweiten Vergleich zu den größten Einzelzentrumskollektiven. Es wurde viel Wert auf die exakte und vollständige Beobachtung aller Patienten im post-interventionellen Zeitraum gelegt. Darüber hinaus wurde die Prozedur von einem erfahrenen kleinen Team (2 Operateure, 2 Echokardiographie-Untersucher) durchgeführt. Die Nachbeobachtungszeit ist natürlich relativ 
gering in Anbetracht der Zeiträume, die für die Mitralklappen-Chirurgie schon veröffentlicht wurden. Bei MitraClip® handelt sich jedoch noch um ein relativ junges Verfahren.

Die Ergebnisse dieser Arbeit könnten Hinweise auf das ideale Patientenkollektiv für MitraClip® geben. Die aktuellen Leitlinien geben bei sekundärer Mitralinsuffizienz keine eindeutige Empfehlung für eine konventionelle Operation, wenn der Patient keine zusätzliche Indikation zur operativen Myokardrevaskularisation hat. In unserem Kollektiv konnten wir keinen signifikanten prognostischen Unterschied zwischen Patienten mit primärer und sekundärer Mitralinsuffizienz feststellen. Auch die verringerte Pumpfunktion des linken Ventrikels scheint für das Outcome nach MitraClip® von geringerer Bedeutung zu sein als für einen konventionellen herzchirurgischen Eingriff. Zwar war dieser Parameter in der univarianten Analyse signifikant mit herzinsuffizienzbedingter Rehospitalisierung assoziiert, es konnte jedoch kein Einfluss auf die Mortalität nach MitraClip®-Intervention festgestellt werden. Allerdings sollte im Vorhinein gut abgewogen werden, ob die Prozedur auch technisch erfolgreich durchgeführt werden kann, da die Prognose der Therapieversager außerordentlich schlecht ist. Sowohl für postprozedurale Mortalität als auch herzinsuffizienzbedingte Rehospitalisierung ist die erfolglose Prozedur der stärkste Prädiktor in unserer Analyse. Bei diesen Patienten ist es deshalb wichtig, unmittelbar nach der Prozedur eine schnelle Re-Evaluierung im Heart Team durchzuführen, um so die Möglichkeit weiterer konventioneller oder interventioneller Korrekturmaßnahmen zu überprüfen. Des Weiteren sollte bei diesen Patienten dem postprozedural erhöhten Risiko für kardiale Dekompensationen mit anschließender Rehospitalisierung mit engen Kontrollintervallen Sorge getragen werden.

In Anbetracht der Tatsache, dass Patienten, die nicht von der Intervention profitieren, eine schlechte Prognose besitzen, stellt sich die Frage nach präprozeduralen Parametern, die die Reparaturfähigkeit der Klappe mittels MitraClip® schon im Vorhinein beurteilen können. In der EVEREST-I-Studie wurden hierfür schon verschiedene anatomische Kriterien aufgestellt. Im Göttinger Kollektiv gibt es Anzeichen dafür, dass Patienten außerhalb der EVERESTKriterien häufiger hospitalisiert werden. Außerdem befand sich der Großteil (91\%) der Therapieversagergruppe außerhalb der EVEREST-Kriterien. In sehr erfahrenen Zentren spielen die EVEREST-Kriterien möglicherweise eine geringere Rolle in Bezug auf die Ergebnisse (Rudolph et al. 2011), wobei wahrscheinlich die Lernkurve eine wichtige Rolle spielt (Schillinger et al. 2011). Zumindest zu Beginn der Etablierung der MitraClip®-Therapie an einem Zentrum stellen die EVEREST-Kriterien aber offenbar ein nützliches Instrument zur Patientenauswahl dar. 
Interessanterweise unterscheiden sich die beiden Gruppen der erfolgreich behandelten und der nicht erfolgreich behandelten Patienten zwar nicht hinsichtlich der Komorbiditäten, jedoch gibt es große Unterschiede in den zum Aufnahmezeitpunkt ermittelten Echoparametern. Ein großer Anteil der Patienten, die nicht erfolgreich behandelt werden konnten, litt nicht nur unter einer schwersten Mitralinsuffizienz (Grad 4+; 86,4\%; n=19 von 22), sondern wies auch signifikant größere linksventrikuläre Diameter und Volumina als die erfolgreich operierten Patienten auf. Diese Tatsache ist nicht weiter verwunderlich, da die Prozedur durch diese linksventrikulären Verhältnisse deutlich schwieriger wird und somit auch häufiger nicht zufriedenstellend beendet werden kann. Jedoch wäre es falsch zu behaupten, dass Patienten mit großen linksventrikulären Diametern und Volumina grundsätzlich für die MitraClip®-Implantation nicht geeignet sind und unausweichlich eine schlechtere Prognose besitzen. Lediglich der LVESD > 55mm war in der univarianten Analyse hinsichtlich der Rehospitalisierung signifikant. Schwierige anatomische Verhältnisse (wie zum Beispiel ein vergrößerter linker Ventrikel) erschweren und verlängern die Prozedur und sollten deswegen erfahrenen Zentren und Teams vorbehalten sein, um ein optimales Outcome zu gewährleisten. Diesen Patienten grundsätzlich die MitraClip®-Therapie vorzuenthalten, ist nach den vorliegenden Ergebnissen falsch, da es keinen Hinweis darauf gibt, dass diese Patienten per se ein schlechtes Outcome haben. Im Göttinger Kollektiv fanden sich allerdings signifikant mehr prozedurale Versagen unter den ersten 50 Fällen, was den Einfluss der Lernkurve verdeutlicht und impliziert, dass Patienten mit schwierigen anatomischen Verhältnissen besser zu einem späteren Zeitpunkt der Lernkurve behandelt werden sollten. Weiterhin sollte man bedenken, dass zwar auch jede Vollnarkose und Beatmungstherapie, wie sie während der Prozedur benötigt wird, bei diesen schwer herzinsuffizienten Patienten ein zusätzliches Risiko bedeutet, jedoch bei diesen Patienten die MitraClip®-Therapie häufig die letzte Therapieoption überhaupt ist.

Die Relevanz der Beobachtung, dass präprozedural hochsymptomatische Patienten im NYHA-Stadium IV eine erhöhte Wahrscheinlichkeit haben zu versterben, ist schwierig zu beurteilen. Die Patienten sollten vor Clip-Implantation medikamentös optimal eingestellt werden, um eine so hochgradige Symptomatik und die damit verbundene schlechtere Prognose möglichst zu vermeiden. Liegt trotz optimaler Medikation ein Stadium NYHA IV vor, sollte der Patient über das hohe Risiko aufgeklärt werden und aufmerksam und in engen Zeitintervallen nachuntersucht werden. NYHA IV ist ein Zeichen einer weit fortgeschrittenen Herzinsuffizienz, sodass man anhand der vorliegenden Ergebnisse zu dem Schluss kommen kann, dass diese Patienten möglichst schon in früheren Stadien der Herzinsuffizienz einer chirurgischen oder interventionellen Therapie zugeführt werden sollten. 
Ein weiterer Effekt der erfolgreichen Therapie mit MitraClip ${ }^{\circledR}$ war die Verringerung der herzinsuffizienzbedingten Krankenhausaufenthalte in diesem Hochrisikokollektiv innerhalb eines Jahres. Es gab folglich bei diesen Patienten deutlich weniger schwere Dekompensationen der Herzinsuffizienz, die eine Hospitalisierung verursachten. Natürlich könnte hier spekuliert werden, dass durch die mindestens halbjährlichen Kontrolluntersuchungen nach Clip-Implantation eine gute Herzinsuffizienztherapie gewährleistet wurde, was möglicherweise Dekompensationen verhindert haben könnte. Allerdings wurden die nicht erfolgreich behandelten Patienten ebenfalls in den gleichen Abständen von uns kontrolliert. Außerdem könnte argumentiert werden, dass die Gruppe, die nicht erfolgreich behandelt werden konnte, ein deutlich höheres Risikoprofil besaß und deswegen auch im Jahr vor MitraClip®Implantation öfter hospitalisiert wurde. Innerhalb der Baseline-Charakteristika gibt es aber keinen Anhalt für diese These. Die einzigen signifikanten Unterschiede zwischen diesen Gruppen waren die pulmonale Hypertonie und das Vorhandensein von Ausschlusskriterien für die EVEREST-II-Studie. Diese genannten Kriterien wurden in dieser Arbeit schon besprochen, und die pulmonale Hypertonie allein zeigte in den uni- und multivarianten Analysen keine Signifikanz.

Für die Zukunft sind große randomisierte Studien unerlässlich, um den Stellenwert der MitraClip®-Therapie zu prüfen. Nach EVEREST II gab es bisher keine weiteren randomisierten Studien, und die Daten der europäischen Register zeigen, dass es sich (verglichen mit den EVEREST-Studienpatienten) im europäischen Raum unter realen Bedingungen um andere Patientenkollektive handelt. Der Hersteller des MitraClip®, Abbott (Abbott Park, Illinois, USA) wird nun mit der RESHAPE-HF-Studie die MitraClip®-Therapie in Kombination mit der aktuellen medikamentösen Standardbehandlung gegenüber der alleinigen medikamentösen Therapie vergleichen. Diese Studie wird als eine prospektive, multizentrische, im 1:1 Verhältnis randomisierte Studie angelegt und ist bereits in einigen europäischen Zentren initiiert worden.

\subsection{FAZIT}

Den größten Einfluss auf das Outcome der mittels MitraClip ${ }^{\circledR}$ behandelten Patienten zeigte in unseren Analysen das akute prozedurale Versagen. Als Implikation für den klinischen Alltag sollte im Vorfeld der MitraClip®-Implantation ein besonderes Augenmerk auf das Screening-Verfahren gelegt werden, und diese Patienten sollten ausführlich im Heart Team besprochen werden, um die passende Therapie individuell für jeden Patienten zu finden. 
Weiterhin konnte festgestellt werden, dass der (für herzchirurgische Eingriffe entwickelte) STS-Score mit einem Cut-off Wert von $12 \%$ gut geeignet ist, um die mittelfristige Mortalität nach der MitraClipß-Prozedur vorherzusagen. 


\section{ZUSAMMENFASSUNG}

Hintergrund: Prädiktoren für die Prognose nach perkutaner Mitralklappenreparatur mit MitraClip $\AA$ sind noch weitgehend unerforscht.

Methoden: In einem Kollektiv von 150 konsekutiven Hochrisiko-Patienten, die von April 2009 bis Juni 2012 mit MitraClip® behandelt wurden, haben wir mit der Kaplan-Meier-Methode Überlebenszeitanalysen zu Gesamtmortalität sowie Rehospitalisierung für BaselineCharakteristika, Risikoscores sowie das akute prozedurale Versagen (definiert als persistierende MI Grad 3 oder 4 bei Entlassung) durchgeführt. Nach univariater Analyse signifikante Prädiktoren wurden mittels eines Cox Proportional Hazards Model multivariat getestet.

Ergebnisse: Das Gesamtkollektiv wies ein Durchschnittsalter von $74 \pm 9$ Jahren sowie ein hohes operatives Risiko auf (log. EuroSCORE $129 \pm 18 \%$, STS-Score 10.5 $\pm 8.7 \%$ ). 66\% der Patienten litten unter einer funktionellen MI. Ein akuter prozeduraler Erfolg konnte bei 85\% erzielt werden. Die Überlebensrate lag nach 30 Tagen bei $96 \%$, nach 1 Jahr bei $79 \%$ und nach 2 Jahren bei 63\%. Der mediane Follow-up-Zeitraum betrug dabei 274 Tage. Die Patienten berichteten über eine deutliche Symptomlinderung, was nach 12 Monaten durch eine Verbesserung des Minnesota Living with Heart Failure Scores um 19.5 Punke $(P<0.001)$ sowie durch eine Verbesserung der Gehstrecke im 6-min-Gehtest um $67.5 \mathrm{~m}$ $(P=0.02)$ objektiviert werden konnte. Echokardiographisch konnte nach 12 Monaten eine signifikante Reduktion des linksventrikulären end-diastolischen Diameters ( $P=0.013)$ sowie des end-diastolischen Volumens $(\mathrm{P}<0.0001)$ nachgewiesen werden.

Nach multivariater Analyse konnten nur das akute prozedurale Versagen (HR 2.73, $\mathrm{P}=0.005)$, NYHA-Stadium IV vor Clip-Implantation (HR 2.12, $\mathrm{P}=0.02$ ) sowie ein STS-Score $\geq 12$ (HR 2.07, $P<0.0001$ ) als signifikante unabhängige Prädiktoren der Gesamtmortalität identifiziert werden. Bezüglich der Prädiktion erneuter herzinsuffizienzbedingter Hospitalisierung war nach multivariater Analyse nur das akute prozedurale Versagen signifikant (HR 2.69, $\mathrm{P}=0.004)$.

\section{Zusammenfassung:}

Patienten, bei denen ein akutes prozedurales Versagen auftrat, hatten signifikant größere und volumenüberladenere Ventrikel, litten häufiger unter einer MI Grad IV sowie unter einer pulmonalen Hypertonie und wiesen häufiger Ausschlusskriterien für die EVEREST-II-Studie 
auf. Weiterhin fanden sich signifikant mehr prozedurale Versagen unter den ersten 50 Fällen, was den Einfluss der Lernkurve verdeutlicht.

In unserem Patientenkollektiv hatte das akute prozedurale Versagen den größten negativen Einfluss auf den Langzeitverlauf, das daher unter allen Umständen verhindert werden muss. Dies könnte insbesondere durch ein rigoroses Patientenscreening erreicht werden. Dabei scheinen die EVEREST-II-Ausschlusskriterien insbesondere zu Beginn der Lernkurve ein nützliches Instrument zur Patientenauswahl zu sein.

Schlüsselwörter: MitraClip®, Perkutane Mitralklappenreparatur, Mitralklappeninsuffizienz, Herzinsuffizienz

Teile dieser Arbeit wurden in der Fachzeitschrift „Eurointervention“ veröffentlicht.

Puls M, Tichelbäcker T, Bleckmann A, Hünlich M, von der Ehe K, Beuthner BE, Rüter K, Beißbarth T, Seipelt R, Schöndube F, Hasenfuß G, Schillinger W. Failure of acute procedural success predicts adverse outcome after percutaneous edge-to-edge mitral valve repair with MitraClip. Eurolntervention 2013 Oct 30. doi:pii: 20130511-02. [Epub ahead of print] 


\section{LITERATURVERZEICHNIS}

(1993): An International Randomized Trial Comparing Four Thrombolytic Strategies for Acute Myocardial Infarction. N Engl J Med 329, 673-682

American Thoracic Society (2002): ATS statement: guidelines for the six-minute walk test. Am J Respir Crit Care Med 166, 111-117

Boekstegers P, Hausleiter J, Baldus S, Bardeleben RS von, Beucher H, Butter C, Franzen O, Hoffmann R, Ince H, Kuck KH et al. (2013): Percutaneous interventional mitral regurgitation treatment using the Mitra-Clip system. Clin Res Cardiol [Epub ahead of print]

Bursi F, Enriquez-Sarano M, Jacobsen SJ, Roger VL (2006): Mitral regurgitation after myocardial infarction: a review. Am J Med 119, 103-112

Bursi F, Barbieri A, Grigioni F, Reggianini L, Zanasi V, Leuzzi C, Ricci C, Piovaccari G, Branzi A, Modena MG (2010): Prognostic implications of functional mitral regurgitation according to the severity of the underlying chronic heart failure: a long-term outcome study. Eur J Heart Fail $\underline{12}, 382-388$

Camm AJ, Lüscher TF, Serruys PW: The ESC textbook of cardiovascular medicine; Blackwell Pub./European Society of Cardiology, Malden, Mass. , Oxford 2006

Camm AJ, Kirchhof P, Lip GYH, Schotten U, Savelieva I, Ernst S, van Gelder IC, Al-Attar N, Hindricks G, Prendergast B et al. (2010): Guidelines for the management of atrial fibrillation: The Task Force for the Management of Atrial Fibrillation of the European Society of Cardiology (ESC). Eur Heart J $\underline{31}$, 2369-2429

Carpentier A (1983): Cardiac valve surgery--the "French correction". J Thorac Cardiovasc Surg $\underline{86}$, 323-337

Ciarka A, Braun J, Delgado V, Versteegh M, Boersma E, Klautz R, Dion R, Bax JJ, van de Veire N (2010): Predictors of mitral regurgitation recurrence in patients with heart failure undergoing mitral valve annuloplasty. Am J Cardiol 106, 395-401

Cohn LH, Couper GS, Aranki SF, Rizzo RJ, Kinchla NM, Collins JJ (1994): Long-term results of mitral valve reconstruction for regurgitation of the myxomatous mitral valve. J Thorac Cardiovasc Surg $\underline{107}$, 143-50; discussion 150-1

Deutsche Akkreditierungsstelle: Anlage zur Akkreditierungsurkunde D-PL-13161-01-00 2011

Enriquez-Sarano M, Akins CW, Vahanian A (2009): Mitral regurgitation. The Lancet $\underline{373}, 1382-1394$

Erdmann E: Klinische Kardiologie: Krankheiten des Herzens, des Kreislaufs und der herznahen Gefässe, 8. Auflage; Springer-Verlag, Berlin, Heidelberg 2011

evalve IMPC: Gebrauchsanweisung MitraClip System 2011

Feldman T, Wasserman HS, Herrmann HC, Gray W, Block PC, Whitlow P, St. Goar F, Rodriguez L, Silvestry F, Schwartz A et al. (2005): Percutaneous Mitral Valve Repair Using the Edge-to-Edge Technique. J Am Coll Card $\underline{46}, 2134-2140$

Feldman T, Kar S, Rinaldi M, Fail P, Hermiller J, Smalling R, Whitlow PL, Gray W, Low R, Herrmann $\mathrm{HC}$ et al. (2009): Percutaneous mitral repair with the MitraClip system: safety and midterm durability in the initial EVEREST (Endovascular Valve Edge-to-Edge REpair Study) cohort. J Am Coll Cardiol 54, 686-694

Feldman T, Foster E, Glower DD, Glower DG, Kar S, Rinaldi MJ, Fail PS, Smalling RW, Siegel R, Rose GA et al. (2011): Percutaneous repair or surgery for mitral regurgitation. N Engl J Med $\underline{364}$, 1395-1406

Foster E, Wasserman HS, Gray W, Homma S, Di Tullio MR, Rodriguez L, Stewart WJ, Whitlow P, Block P, Martin R et al. (2007): Quantitative assessment of severity of mitral regurgitation by serial echocardiography in a multicenter clinical trial of percutaneous mitral valve repair. Am J Cardiol $\underline{100}$, 1577-1583 
Geissler HJ, Hölzl P, Marohl S, Kuhn-Régnier F, Mehlhorn U, Südkamp M, Vivie ER de (2000): Risk stratification in heart surgery: comparison of six score systems. Eur J Cardiothorac Surg 17, 400-406

Glower DD (2012): Surgical approaches to mitral regurgitation. J Am Coll Cardiol $\underline{60}, 1315-1322$

Grigioni F, Enriquez-Sarano M, Zehr KJ, Bailey KR, Tajik AJ (2001): Ischemic mitral regurgitation: long-term outcome and prognostic implications with quantitative Doppler assessment. Circulation $\underline{103}$, 1759-1764

Hamm CW, Albrecht A, Bonzel T, Kelm M, Lange H, Schächinger V, Terres W, Voelker W (2008): Diagnostische herzkatheteruntersuchung.[sic!] Clin Res Cardiol 97, 475-512

lung B, Baron G, Butchart EG, Delahaye F, Gohlke-Bärwolf C, Levang OW, Tornos P, Vanoverschelde J, Vermeer $F$, Boersma $E$ et al. (2003): A prospective survey of patients with valvular heart disease in Europe: The Euro Heart Survey on Valvular Heart Disease Eur Heart J $\underline{24}, 1231-$ 1243

Maisano F, Franzen O, Baldus S, Schäfer U, Hausleiter J, Butter C, Ussia GP, Sievert H, Richardt G, Widder JD et al. (2013): Percutaneous Mitral Valve Interventions in the Real World: Early and One Year Results From the ACCESS-EU, a Prospective, Multicenter, Non-Randomized Post-Approval Study of the MitraClip ${ }^{\circledR}$ Therapy in Europe. J Am Coll Card

MAISEL A, MUELLER C, ADAMSJR K, ANKER S, ASPROMONTE N, CLELAND J, COHENSOLAL A, DAHLSTROM U, DEMARIA A, DISOMMA S (2008): State of the art: Using natriuretic peptide levels in clinical practice. Eur J Heart Fail 10, 824-839

Mauri L, Garg P, Massaro JM, Foster E, Glower D, Mehoudar P, Powell F, Komtebedde J, McDermott E, Feldman T (2010): The EVEREST II Trial: design and rationale for a randomized study of the evalve mitraclip system compared with mitral valve surgery for mitral regurgitation. Am Heart J $\underline{160}$, 23-29

Morgan K, McGee H, Shelley E (2007): Quality of life assessment in heart failure interventions: a 10year (1996-2005) review. Eur J Cardiovasc Prev Rehabil 14, 589-607

Nkomo VT, Gardin JM, Skelton TN, Gottdiener JS, Scott CG, Enriquez-Sarano M (2006): Burden of valvular heart diseases: a population-based study. Lancet $\underline{368}, 1005-1011$

Paranskaya L, D'Ancona G, Bozdag-Turan I, Akin I, Kische S, Turan GR, Divchev D, Rehders TC, Schneider H, Ortak J et al. (2012): Early and mid-term outcomes of percutaneous mitral valve repair with the MitraClip®: comparative analysis of different EuroSCORE strata. Eurolntervention $\underline{8}, 571-578$

Paranskaya L, D'Ancona G, Bozdag-Turan I, Akin I, Kische S, Turan GR, Rehders T, Ortak J, Nienaber CA, Ince H (2012): Residual mitral valve regurgitation after percutaneous mitral valve repair with the mitraclip ${ }^{\circledR}$ system is a risk factor for adverse one-year outcome. Cathet Cardiovasc Intervent, $\mathrm{n} / \mathrm{a}$

Pleger ST, Mereles D, Schulz-Schönhagen M, Krumsdorf U, Chorianopoulos E, Rottbauer W, Katus HA, Bekeredjian R (2011): Acute safety and 30-day outcome after percutaneous edge-to-edge repair of mitral regurgitation in very high-risk patients. Am J Cardiol 108, 1478-1482

Rudolph V, Knap M, Franzen O, Schlüter M, Vries T de, Conradi L, Schirmer J, Treede H, Wegscheider K, Costard-Jäckle A et al. (2011): Echocardiographic and Clinical Outcomes of MitraClip Therapy in Patients Not Amenable to Surgery. J Am Coll Card $\underline{58}$, 2190-2195

Schillinger W, Kerl S: Standard Operating Procedure MitraClip V2 2011

Schillinger W, Athanasiou T, Weicken N, Berg L, Tichelbäcker T, Puls M, Hünlich M, Wachter R, Helms H, Seipelt R et al. (2011): Impact of the learning curve on outcomes after percutaneous mitral valve repair with MitraClip and lessons learned after the first 75 consecutive patients. Eur $\mathrm{J}$ Heart Fail $\underline{13}, 1331-1339$

Schillinger W, Hünlich M, Baldus S, Ouarrak T, Boekstegers P, Hink U, Butter C, Bekeredjian R, Plicht $\mathrm{B}$, Sievert $\mathrm{H}$ et al. (2013): Acute outcomes after MitraClip therapy in highly aged patients: results from the German TRAnscatheter Mitral valve Interventions (TRAMI) Registry. Eurolntervention $\underline{9}, 84-90$

Schmidt, R. F. (Hrsg.) (2007): Physiologie des Menschen: Mit Pathophysiologie ; mit 77 Tabellen ; [+ IMPP-Fragen Physiologie online], 30. Auflage; Springer, Heidelberg 2007 
Tribouilloy C, Grigioni F, Avierinos JF, Barbieri A, Rusinaru D, Szymanski C, Ferlito M, Tafanelli L, Bursi F, Trojette $F$ et al. (2009): Survival implication of left ventricular end-systolic diameter in mitral regurgitation due to flail leaflets a long-term follow-up multicenter study. J Am Coll Cardiol 54, 19611968

Vahanian A, Alfieri O, Andreotti F, Antunes MJ, Barón-Esquivias G, Baumgartner H, Borger MA, Carrel TP, Bonis M de, Evangelista A et al. (2012): Guidelines on the management of valvular heart disease (version 2012): The Joint Task Force on the Management of Valvular Heart Disease of the European Society of Cardiology (ESC) and the European Association for Cardio-Thoracic Surgery (EACTS). Eur J Cardiothorac Surg 느, S1-S44

Wendt D, Osswald BR, Kayser K, Thielmann M, Tossios P, Massoudy P, Kamler M, Jakob H (2009): Society of Thoracic Surgeons score is superior to the EuroSCORE determining mortality in high risk patients undergoing isolated aortic valve replacement. Ann Thorac Surg $\underline{88}, 468-74$; discussion 474-5 


\section{ABBILDUNGSVERZEICHNIS}

Abbildung 1: Ursachen der chronischen Mitralklappeninsuffizienz ............................................... 6

Abbildung 2: Mechanismen der chronischen Mitralklappeninsuffizienz …....................................... 6

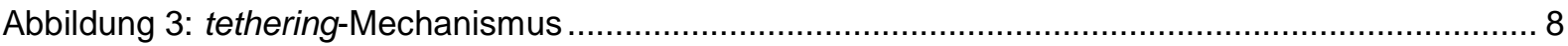

Abbildung 4: Kaplan-Meier-Analyse der Lebenserwartung von Patienten nach Myokardinfarkt mit unterschiedlichen Schweregraden einer ischämischen Mitralinsuffizienz ....................................... 10

Abbildung 5: schematische Darstellungen der Mitralklappe mit Pathologien und operativen

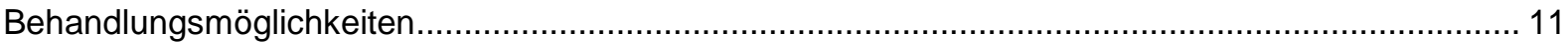

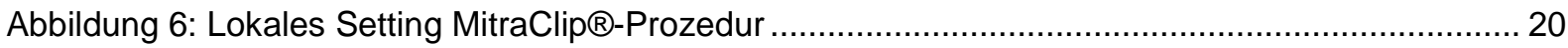

Abbildung 7: 3D-TEE, Aufsicht auf die Mitralklappe in der Diastole ................................................. 21

Abbildung 8: 3D-TEE, Aufsicht auf die Mitralklappe in der Systole .................................................. 21

Abbildung 9: Deutlich sichtbarer exzentrischer Jet an der Wand des linken Vorhofs......................... 21

Abbildung 10: Platzierung und Ausrichtung des geöffneten MitraClip ${ }^{\circledR}$ an der Stelle des

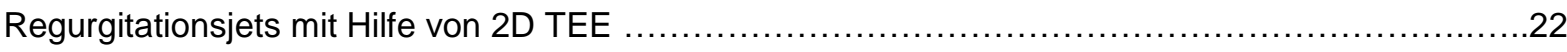

Abbildung 11: Platzierung und Ausrichtung des geöffneten MitraClip ${ }^{\circledR}$ an der Stelle des

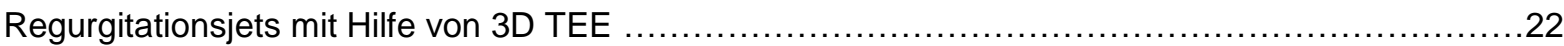

Abbildung 12: double-orifice der Mitralklappe nach Clip-Implantation ................................................ 23

Abbildung 13: Ergebnis der perkutanen Mitralklappenreparatur post-interventionell ..................24

Abbildung 14: Ergebnis der perkutanen Mitralklappenreparatur nach einem Jahr...................24

Abbildung 15: 6- und 12-Monatsdaten der Follow-Up-Untersuchungen ........................................... 31

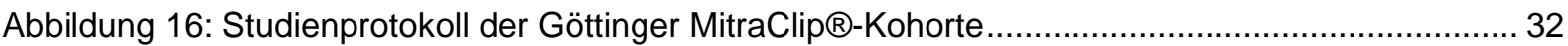

Abbildung 17: Kaplan-Meier-Kurven der Gesamtmortalität im Follow-up in Abhängigkeit vom Erreichen

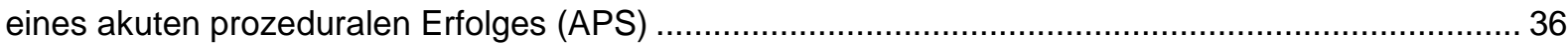

Abbildung 18: Kaplan-Meier-Kurven der herzinsuffizienzbedingten erneuten Hospitalisierung in Abhängigkeit vom Erreichen eines akuten prozeduralen Erfolges (APS) ........................................... 39

Abbildung 19: Anzahl herzinsuffizienzbedingter Hospitalisationen innerhalb eines Jahres vor und nach MitraClip®-Implantation 


\section{ANHANG}

\subsection{Minnesota Living With Heart Failure Questionnaire}

Bitte kreisen Sie bei den folgenden Fragen ein, inwieweit Ihre Herzkrankheit lhr Leben in den letzten 4 Wochen beeinträchtigt hat. Wenn eine Frage nicht auf Sie zutrifft, kreisen Sie bitte die 0 ein.

Nein Selten Immer

1. Traten Schwellungen an den Fưknöcheln, Beinen etc auf?

2. Mussten Sie sich tagsüber hinsetzen oder \begin{tabular}{lllllll} 
hinlegen um sich auszuruhen? & 0 & 1 & 2 & 3 & 4 & 5 \\
\hline
\end{tabular}

3. Hatten Sie Schwierigkeiten beim Umhergehen oder Treppensteigen?

\begin{tabular}{llllll}
0 & 1 & 2 & 3 & 4 & 5 \\
\hline
\end{tabular}

4. Hatten Sie Schwierigkeiten bei Erledigungen im Haus oder im Garten?

$\begin{array}{llllll}0 & 1 & 2 & 3 & 4 & 5\end{array}$

5. Hatten Sie Schwierigkeiten sich von zuhause wegzubewegen?

$\begin{array}{llllll}0 & 1 & 2 & 3 & 4 & 5\end{array}$

6. Hatten Sie Schwierigkeiten nachts gut zu schlafen?

$\begin{array}{llllll}0 & 1 & 2 & 3 & 4 & 5\end{array}$

7. Hatten Sie Schwierigkeiten im Zusammenleben oder gemeinsamen Tätigkeiten mit Freunden oder der Familie?

$\begin{array}{llllll}0 & 1 & 2 & 3 & 4 & 5\end{array}$

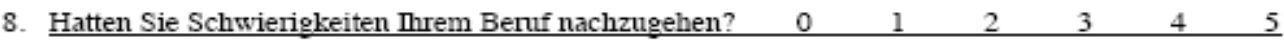

9. Hatten Sie Schwierigkeiten bei Ihrer Ereizeitgestaltung beim Sport oder ihren Hobbys? $\quad \begin{array}{lllllll}0 & 1 & 2 & 3 & 4 & 5\end{array}$

10. Waren Sie bei Thren sexuellen Aktivitäten beeinträchtigt?

$\begin{array}{llllll}0 & 1 & 2 & 3 & 4 & 5\end{array}$

11. Konnten Sie von den Nahrungsmitteln, die Sie \begin{tabular}{llllllll} 
gerne mögen, nur weniger essen? & 0 & 1 & 2 & 3 & 4 & 5 \\
\hline
\end{tabular}

12. Waren Sie kurzatmig? $\quad \begin{array}{lllllll}0 & 1 & 2 & 3 & 4 & 5\end{array}$

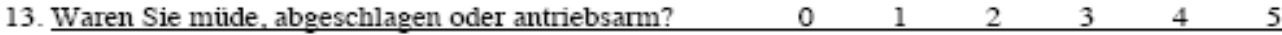

14. Mussten Sie in einem Krankenhaus stationär aufgenommen werden?

\begin{tabular}{llllll}
0 & 1 & 2 & 3 & 4 & 5 \\
\hline
\end{tabular}

15. Mussten Sie wegen Threr Herzerkrankung Geld ausgeben $\begin{array}{llllll}0 & 1 & 2 & 3 & 4 & 5\end{array}$

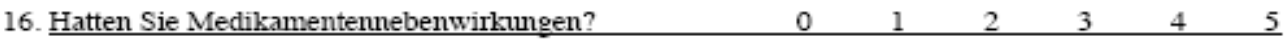

17. Hatten Sie den Eindruck, dass Sie für Thre

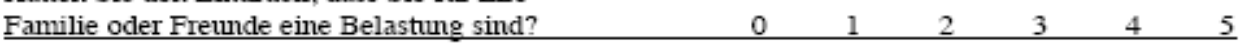

18. Hatten Sie den Eindruck, dass Sie Thr Leben

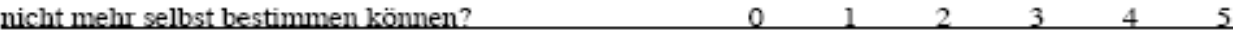

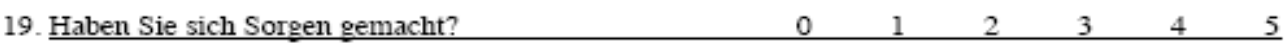

20. Hatten Sie Schwierigkeiten sich zu konzentrieren oder sich an Dinge zu erinnem?

\begin{tabular}{llllll}
0 & 1 & 2 & 3 & 4 & 5 \\
\hline
\end{tabular}

21. Hatten Sie Zeiten, in denen Sie ohne äußeren Anlass traurig und nicht aufzuheitem waren?

$\begin{array}{llllll}0 & 1 & 2 & 3 & 4 & 5\end{array}$




\subsection{Aufnahmebogen}

\section{Aufnahmebogen Göttinger MitraClip -Kohorte}
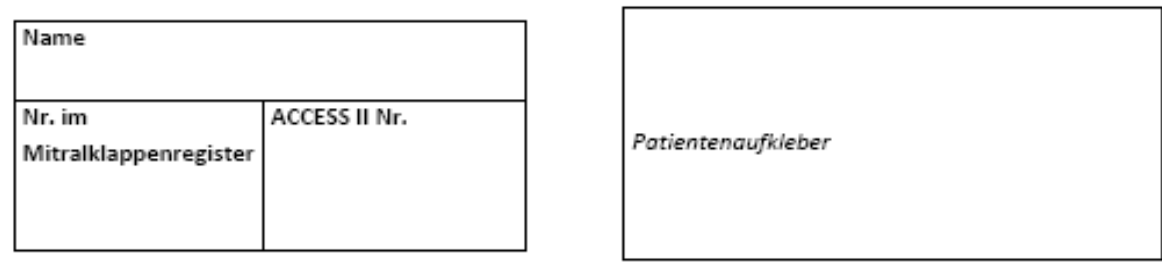

Kontaktdaten

\begin{tabular}{|l|l|}
\hline Telefonnummer & \\
\hline $\begin{array}{l}\text { Telefonnummer Angehörige } \\
\text { (optional) }\end{array}$ & \\
\hline
\end{tabular}

Krankenhausaufenthalte in den letzten 12 Monaten

\begin{tabular}{|l|l|l|}
\hline Zeitpunkt und Dauer & Krankenhaus & Aufnahmegrund \\
\hline & & \\
\hline & & \\
\hline & & \\
\hline
\end{tabular}

NYHA- Klassifikation

MLHFQ

\begin{tabular}{|l|l|l|l|}
\hline I & II & III & IV \\
\hline
\end{tabular}

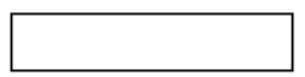

Vitalparameter:

\begin{tabular}{|c|c|c|c|c|c|}
\hline Größe & Gewicht & Temp & $\mathrm{RR}$ & $\mathrm{HF}$ & Resp \\
\hline $\mathrm{cm}$ & $\mathrm{kg}$ & ${ }^{\circ} \mathrm{C}$ & $\mathrm{mmHg}$ & $/ \mathrm{min}$ & $/ \min$ \\
\hline
\end{tabular}

6MWT

\begin{tabular}{|ll|ll|}
\hline+ & $\mathrm{m}$ & $\mathrm{O}=$ & $\mathrm{m}$ \\
\hline
\end{tabular}

BNP und Trop-T nachgemeldet? 


\section{DANKSAGUNG}

Für das Gelingen der vorliegenden Arbeit bin ich einigen Personen zu Dank verpflichtet. An erster Stelle danke ich Herrn Prof. Dr. med. Wolfgang Schillinger für die Vergabe des Themas, die exzellente Betreuung und das ausgezeichnete und motivierende Arbeitsverhältnis. Außerdem danke ich Frau Dr. med. Miriam Puls für Ihre hervorragenden und hilfreichen Korrekturen und für die Hilfestellung beim Erlernen des wissenschaftlichen Arbeitens. Weiterhin möchte ich mich bei dem Team des Echokardiographielabors und den Mitarbeitern der Leitstelle Innere Medizin der Universitätsmedizin Göttingen für die gute Zusammenarbeit bei der Durchführung der Follow-Up-Termine bedanken. Frau Dr. med. Ninja Weicken danke ich für die sehr gute Einarbeitung und Hilfestellung in der Anfangszeit meiner Tätigkeit als Doktorand in der Arbeitsgruppe für Interventionelle Kardiologie. Des Weiteren gilt mein Dank Frau Nina Schaper für die abschließenden formalen Korrekturen. 